\title{
HYPERBOLICITY OF RENORMALIZATION OF CRITICAL CIRGLE MAPS
}

\author{
by Michael YAMPOLSKY
}

The renormalization theory of critical circle maps was developed in the late 1970's-early 1980's to explain the occurence of certain universality phenomena. These phenomena can be observed empirically in smooth families of circle homeomorphisms with one critical point, the so-called critical circle maps, and are analogous to Feigenbaum universality in the dynamics of unimodal maps. In the works of Ostlund et al. [ORSS] and Feigenbaum et al. [FKS] they were translated into hyperbolicity of a renormalization transformation.

The first renormalization transformation in one-dimensional dynamics was constructed by Feigenbaum and independently by Coullet and Tresser in the setting of unimodal maps. The recent spectacular progress in the unimodal renormalization theory began with the seminal work of Sullivan [Sull,Sul2,MvS]. He introduced methods of holomorphic dynamics and Teichmüller theory into the subject, developed a quadratic-like renormalization theory, and demonstrated that renormalizations of unimodal maps of bounded combinatorial type converge to a horseshoe attractor. Subsequently, McMullen [McM2] used a different method to prove a stronger version of this result, establishing, in particular, that renormalizations converge to the attractor at a geometric rate. And finally, Lyubich [Lyu4,Lyu5] constructed the horseshoe for unbounded combinatorial types, and showed that it is uniformly hyperbolic, with one-dimensional unstable direction, thereby bringing the unimodal theory to a completion.

The renormalization theory of circle maps has developed alongside the unimodal theory. The work of Sullivan was adapted to the subject by de Faria, who constructed in $[\mathrm{dF} 1, \mathrm{dF} 2]$ the renormalization horseshoe for critical circle maps of bounded type. Later de Faria and de Melo [dFdM2] used McMullen's work to show that the convergence to the horseshoe is geometric. The author in [Yal,Ya2] demonstrated the existence of the horseshoe for unbounded types, and studied the limiting situation arising when the combinatorial type of the renormalization grows without a bound.

Despite the similarity in the development of the two renormalization theories up to this point, the question of hyperbolicity of the horseshoe attractor presents a notable difference. Let us recall without going into details the structure of the argument given by Lyubich in the unimodal case. The first part of Lyubich's work was to to endow the

This work was partially supported by NSF Grant DMS-9804606. 
ambient space of the renormalization transformation with the structure of a complexanalytic manifold, with respect to which renormalization is an analytic operator. $\mathrm{He}$ then showed that the stable sets of periodic points of this operator are codimension one submanifolds and used an argument based on McMullen's Tower Rigidity Theorem and an infinite dimensional version of the Schwarz Lemma to show that renormalization is a strict contraction in the stable direction. The second part of Lyubich's work was to show the existence of an unstable direction. This is a fundamentally more difficult part of the proof, based on Lyubich's Rigidity Theorem [Lyu3] and an elegant argument relating non-hyperbolicity of the renormalization operator to certain topological properties of its orbits.

In contrast, in the case of critical circle maps it has been well-known how one may construct an unstable direction for the renormalization. The difficulty arises with the first part of the program. There is no obvious way to turn the renormalization into an analytic operator. Indeed, the definition of renormalization for critical circle maps essentially employs real symmetry, and resists complexification. To circumvent this obstacle, in this paper we introduce a different renormalization construction, a renormalization of critical cylinder maps. This construction is strongly motivated by a so-called parabolic renormalization, a degenerate case of renormalization, which we studied in detail in [Ya2]. There is a natural functorial relation between the renormalization of cylinder maps and the usual one which allows us to transfer the results back and forth. By virtue of its construction, the cylinder renormalization is an analytic operator on a Banach manifold. We then show that the stable sets of the periodic orbits of this operator are codimension one submanifolds, and prove that every such orbit is hyperbolic. Our result gives a rigorous mathematical explanation of the universality phenomena, settling this well-studied problem.

The structure of the paper is as follows. In $\S 1$ we introduce critical circle maps. We discuss the universality phenomena and define renormalization in $\S 2$. In $\S 4$ we formulate the Renormalization Hyperbolicity Conjecture (Lanford's Program) and state our results. In the following section we discuss some of the constructions from de Faria's and our own earlier work. In $\S 6$ we introduce parabolic renormalization, mainly as a motivation for futher discussion. We then define the cylinder renormalization operator in $\S 7$ and proceed to prove our main theorems.

\section{Acknowledgements}

I had many fruitful discussions with Adam Epstein on the subject of parabolic renormalization of critical circle maps and related topics, and I wish to thank him for generously sharing his mathematical insights with me. I am also grateful to Folkert Tangerman for explaining to me the construction of the unstable direc- 
tion some years ago. I would like to express my gratitude to Mikhail Lyubich for the many conversations we had as this paper developed, for his advice, patience, and constant encouragement. I want to thank Welington de Melo for the helpful criticism of an earlier version of this work. The bulk of this work was conducted at the Yale Mathematics Department, and I wish to thank my colleagues there for making my postdoctoral experience so enjoyable. My special thanks go to Peter Jones for his guidance. Thanks are also due to IHES where this paper was completed. Finally, I wish to thank the referee for the helpful comments.

\section{Preliminaries}

Some notations. - We use dist and diam to denote the Euclidean distance and diameter in $\mathbf{C}$. We shall say that two real numbers A and B are K-commensurable for $\mathrm{K}>1$ if $\mathrm{K}^{-1}|\mathrm{~A}| \leq|\mathrm{B}| \leq \mathrm{K}|\mathrm{A}|$. The notation $\mathrm{D}_{r}(z)$ will stand for the Euclidean disk with the center at $z \in \mathbf{G}$ and radius $r$. The unit disk $\mathrm{D}_{1}(0)$ will be denoted $\mathbf{D}$. The plane $(\mathbf{G} \backslash \mathbf{R}) \cup \mathrm{J}$ with the parts of the real axis not contained in the interval $\mathrm{J} \subset \mathbf{R}$ removed will be denoted $\mathbf{G}_{\mathrm{J}}$. By the circle $\mathbf{T}$ we understand the affine manifold $\mathbf{R} / \mathbf{Z}$, it is naturally identified with the unit circle $\mathrm{S}^{1}=\partial \mathbf{D}$. The real translation $x \mapsto x+\theta$ projects to the rigid rotation by angle $\theta, \mathbf{R}_{\theta}: \mathbf{T} \rightarrow \mathbf{T}$. For two points $a$ and $b$ in the circle $\mathbf{T}$ which are not diametrically opposite, $[a, b]$ will denote the shorter of the two arcs connecting them. As usual, $|[a, b]|$ will denote the length of the arc. For two points $a, b \in \mathbf{R},[a, b]$ will denote the closed interval with endpoints $a, b$ without specifying their order. The cylinder in this paper, unless otherwise specified will mean the affine manifold $\mathbf{C} / \mathbf{Z}$. Its equator is the circle $\{\operatorname{Im} z=0\} / \mathbf{Z} \subset \mathbf{G} / \mathbf{Z}$. A topological annulus $\mathrm{A} \subset \mathbf{G} / \mathbf{Z}$ will be called an equatorial annulus, or an equatorial neighborhood, if it has a smooth boundary and contains the equator.

By "smooth" in this paper we will mean "of class C'", unless another degree of smoothness is specified. The notation " $\mathrm{C} \omega$ " will stand for "real-analytic".

Critical circle maps. - A critical circle map is an orientation preserving automorphism of $\mathbf{T}$ of class $\mathrm{C}^{3}$ with a single critical point $c$. A further assumption is made that the critical point is of cubic type. This means that for a lift $\bar{f}: \mathbf{R} \rightarrow \mathbf{R}$ of a critical circle map $f$ with critical points at the integer translates of $\bar{c}$,

$$
\bar{f}(x)-\bar{f}(\bar{c})=(x-\bar{c})^{3}(\text { const }+\mathrm{O}(x-\bar{c})) .
$$

We note that all the renormalization results will hold true if in the above definition " 3 " as the order of smoothness and the order of the critical point is replaced by any other odd number. To fix our ideas, we will always place the critical point of $f$ at $0 \in \mathbf{T}$. 
Being a homeomorphism of the circle, a critical circle map $f$ has a welldefined rotation number, denoted $\rho(f)$. It is useful to represent $\rho(f)$ as a contined fraction with positive terms

$$
\rho(f)=\frac{1}{r_{0}+\frac{1}{r_{1}+\frac{1}{r_{2}+\cdots}}}
$$

Further on we will abbreviate this expression as $\left[r_{0}, r_{1}, r_{2}, \ldots\right]$ for typographical convenience. Note that the numbers $r_{i}$ are determined uniquely if and only if $\rho(f)$ is irrational. In this case we shall say that $\rho(f)$ (or $f$ itself) is of the type bounded by $\mathrm{B}$ if $\sup r_{i} \leq \mathrm{B}$; it is of a periodic type if the sequence $\left\{r_{i}\right\}$ is periodic. Recall that an iterate $f^{k}(0)$ is called a closest return of the critical point if the arc $\left[0, f^{k}(0)\right]$ contains no iterates $f^{i}(0)$ with $i<k$. By a classical result of Poincaré every circle homeomorphism $f$ with an irrational rotation number is semi-conjugate to the rigid rotation $\mathbf{R}_{\rho(f)}$. Yoccoz [Yoc] has shown that in the case when $f$ is a critical circle map. This implies, in particular, that if we denote $\left\{p_{m} / q_{m}\right\}$ the sequence of best rational approximations of $\rho(f)$ obtained as the truncated continued fractions $p_{m} / q_{m}=\left[r_{0}, r_{1}, \ldots, r_{m-1}\right]$, then the iterates $\left\{f^{q_{m}}(0)\right\}$ are closest returns of 0 . Set $\mathrm{I}_{m} \equiv\left[0, f^{q_{m}}(0)\right]$.

An important one-parameter family of examples of critical circle maps, the so-called standard (or Arnold's) maps, is obtained as follows. Given any $\theta \in \mathbf{R}$ the map

$$
\mathrm{A}_{\theta}(x)=x+\theta-\frac{1}{2 \pi} \sin 2 \pi x
$$

commutes with the unit translation: $\mathrm{A}(x+1)=\mathrm{A}(x)+1$. Therefore it has a welldefined projection to an endomorphism of the circle. We denote this endomorphism $f_{\theta}$, although in reality it only depends on the class $\theta \bmod \mathbf{Z}$. An elementary computation shows that every $\mathrm{A}_{\theta}$ is strictly monotone, and has critical points at integer values of $x$, all of cubic type. Therefore, each $f_{\theta}$ is a critical circle mapping. The considerations of monotone dependence on the parameter imply that

$$
\theta \rightarrow \rho(\theta) \equiv \rho\left(f_{\theta}\right)
$$

is a continuous monotone map of $\mathbf{T}$ onto itself. Whenever $t \in \mathbf{T}$ is irrational, $\rho^{-1}(t)$ is a single point. For $t=p / q$ the set $\rho^{-1}(t)$ is a closed interval, for every parameter value in this interval the homeomorphism $f_{\theta}$ has a periodic orbit with a combinatorial rotation number $p / q$. This orbit has eigenvalue one at the two endpoints of the interval. 


\section{Universality and renormalization}

\subsection{The golden-mean universality phenomenon}

The raison d'être of the renormalization theory of critical circle maps is a universality phenomenon numerically observed as early as in mid-1970's in one-parameter families like the Arnold's family described above (see [FKS], [ORSS]).

For $\mu \in \mathbf{T}$ let $f_{\mu}$ be a one-parameter family of critical circle maps whose dependence on the parameter is smooth and strictly monotone, with $\rho\left(f_{0}\right)=0$. Let us denote by $\mathscr{I}(x)$ the set of parameter values $\mu$ with $\rho\left(f_{\mu}\right)=x$. It is not difficult to show that $\mathscr{I}(x)$ is a single point for irrational values of $x$; in the case when $x$ is rational, $\mathscr{I}(x)$ is a closed interval. Consider now the intervals $\mathscr{I}_{n} \equiv$ $\mathscr{I}\left(\left[r_{0}, r_{1}, \ldots, r_{n-1}\right]\right)$ with $r_{0}=r_{1}=\cdots=r_{n-1}=1$. The maps with parameter values in $\mathscr{I}_{n}$ possess periodic orbits with periods $q_{n}$, where $p_{n} / q_{n}$ is the $n$-th convergent of the infinite continued fraction $[1,1,1,1, \ldots]$. The first numerical observation one makes is that there is a particular number $\delta_{*} \approx 2.83361$ such that the length of the interval $\mathscr{I}_{n}$ behaves asymptotically for large $n$ as const $\cdot \delta_{*}^{-n}$. The rate of the geometric decay $\delta$ is universal, that is independent on the chosen one-parameter family $f_{\mu}$. The second observation applies to the limit of the intervals $\mathscr{I}_{n}$, i.e. the parameter value $\mu_{1}$ for which the map $f_{\mu_{1}}$ possesses the golden mean rotation number

$$
\rho\left(f_{\mu_{1}}\right)=\frac{\sqrt{5}-1}{2}=[1,1,1,1, \ldots] .
$$

Denoting by $\lambda_{n}$ the scaling ratio of the length of the closest return arcs

$$
\lambda_{n}=\left|\left[f_{\mu_{1}}^{\circ q_{n}}(c), c\right]\right| /\left|\left[c, f_{\mu_{1}}^{\circ q_{n-1}}(c)\right]\right|,
$$

we observe that $\lambda_{n}$ converge to a constant $\lambda_{*} \approx 0.7760513$. The number $\lambda_{*}$ is again independent of the family.

Let us remark, that if we consider homeomorphisms of the circle with a nonflat critical point of the order other than three, the universal constants will change. Using the terminology of statistical physics, the order of the critical point specifies the universality class.

\subsection{Definition of renormalization of critical circle maps}

The strong analogy with universality phenomena in statistical physics and with the already discovered Feigenbaum-Collett-Tresser universality in unimodal maps, led the authors of [FKS] and [ORSS] to explain the existence of the universal constants by introducing a renormalization operator acting on critical circle maps. The definition is by no means straightforward. We shall arrive at it after 
a brief discussion, which will be helpful in understanding the motivation of the results in this paper.

The general strategy of defining a renormalization of a given dynamical system $\mathrm{F}: \mathrm{X} \rightarrow \mathrm{X}$ is as follows (c.f. [Lyu2]). Select a proper subset $\mathrm{Y}$ of $\mathrm{X}$. For a point $x \in \mathrm{Y}$ whose forward orbit under $\mathrm{F}$ returns to $\mathrm{Y}$ denote $\mathrm{R}_{\mathrm{F}}(x) \in \mathrm{Y}$ the first such return. Provided that it is true for every point in $\mathrm{Y}$, this defines the first return map $\mathrm{R}_{\mathrm{F}}: \mathrm{Y} \rightarrow \mathrm{Y}$. Let $\mathrm{A}: \mathrm{Y} \rightarrow \mathrm{A}(\mathrm{Y})$ be an affine map, such that the size of $\mathrm{A}(\mathrm{Y})$, appropriately understood, is the same as the size of the original space $\mathrm{X}$. The rescaled first return map $\mathrm{A} \circ \mathrm{R}_{\mathrm{F}} \circ \mathrm{A}^{-1}$ is the renormalization of $\mathrm{F}$.

For the role of the set $\mathrm{Y}$ in the case of a critical circle map $f: \mathbf{T} \rightarrow \mathbf{T}$ one takes the union of arcs $\mathrm{A}_{m}=\mathrm{I}_{m} \cup \mathrm{I}_{m+1}$ for some $m>1$, where as before $\mathrm{I}_{m}$ stands for $\left[0, f^{q_{m}}(0)\right]$. This choice was made in [ORSS] and [FKS], and has been followed since, although a different choice would have led to the same results. The first return map $\mathrm{R}_{m}: \mathrm{A}_{m} \rightarrow \mathrm{A}_{m}$ is defined piecewise by $f^{q_{m}}$ on $\mathrm{I}_{m+1}$ and by $f^{q_{m+1}}$ on $\mathbf{I}_{m}$. To view $\mathbf{R}_{m}$ as a critical circle map we may identify the neighborhoods of points $f^{q_{m}}(0)$ and $f^{q_{m+1}}(0)$ by the iterate $f^{q_{m+1}-q_{m}}$. This identification transforms the arc $\mathrm{A}_{m}$ into a $\mathrm{C}^{3}$-smooth closed one-dimensional manifold $\tilde{\mathrm{A}}_{m}, \mathrm{R}_{m}$ projects to a smooth homeomorphism $\tilde{\mathrm{R}}_{m}: \tilde{\mathrm{A}}_{m} \rightarrow \tilde{\mathrm{A}}_{m}$ with a critical point at 0 . However, the manifold $\tilde{\mathrm{A}}_{m}$ does not possess a canonical affine structure; the choice of a smooth identification $\phi: \tilde{\mathrm{A}}_{m} \rightarrow \mathbf{R} / \mathbf{Z}$ gives rise to a plethora of different critical circle maps, all smoothly conjugate.

As we can see from the above discussion, the space of critical circle maps is ill-suited to define renormalization. The authors of [ORSS] and [FKS] circumvented this difficulty by replacing critical circle maps with different objects:

Definition 2.1. - A commuting pair $\zeta=(\eta, \xi)$ consists of two $\mathrm{C}^{3}$-smooth orientation preserving interval homeomorphisms $\eta: \mathrm{I}_{\eta} \rightarrow \eta\left(\mathrm{I}_{\eta}\right), \xi: \mathrm{I}_{\xi} \rightarrow \xi\left(\mathrm{I}_{\xi}\right)$, where

(I) $\mathrm{I}_{\eta}=[0, \xi(0)], \mathrm{I}_{\xi}=[\eta(0), 0]$;

(II) Both $\eta$ and $\xi$ have homeomorphic extensions to interval neighborhoods of their respective domains with the same degree of smoothness, which commute, $\eta \circ \xi=\xi \circ \eta$;

(III) $\xi \circ \eta(0) \in \mathrm{I}_{\eta}$;

(IV) $\eta^{\prime}(x) \neq 0 \neq \xi^{\prime}(y)$, for all $x \in \mathrm{I}_{\eta} \backslash\{0\}$, and all $y \in \mathrm{I}_{\xi} \backslash\{0\}$.

The commutation condition allows one to iterate the extensions of the maps of a commuting pair. We can also use it to repeat the glueing construction. That is, given a critical commuting pair $\zeta=(\eta, \xi)$ we can regard the interval $\mathrm{I}=$ $[\eta(0), \xi \circ \eta(0)]$ as a circle, identifying $\eta(0)$ and $\xi \circ \eta(0)$ and define $f_{\zeta}: \mathrm{I} \rightarrow$ I by

$$
f_{\zeta}=\left\{\begin{array}{l}
\eta \circ \xi(x) \text { for } x \in[\eta(0), 0] \\
\eta(x) \text { for } x \in[0, \xi \circ \eta(0)]
\end{array} .\right.
$$




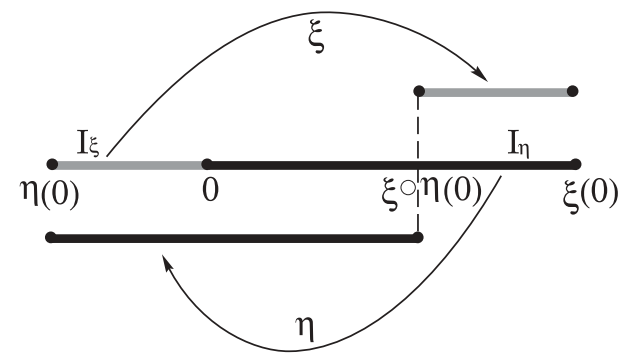

FIG. 1. - A commuting pair

The mapping $\xi$ extends to a $\mathrm{C}^{3}$-diffeomorphism of open neighborhoods of $\eta(0)$ and $\xi \circ \eta(0)$. Using it as a local chart we turn the interval I into a closed onedimensional manifold M. Condition (II) above implies that the mapping $f_{\zeta}$ projects to a well-defined $\mathrm{C}^{3}$-smooth homeomorphism $\mathrm{F}_{\zeta}: \mathrm{M} \rightarrow \mathrm{M}$. Identifying $\mathrm{M}$ with the circle by a diffeomorphism $\phi: \mathrm{M} \rightarrow \mathbf{T}$ we recover a critical circle mapping $f^{\phi}=\phi \circ \mathrm{F}_{\zeta} \circ \phi^{-1}$. The critical circle mappings corresponding to two different choices of $\phi$ are conjugated by a diffeomorphism, and thus we recovered a $\mathrm{C}^{3}$-smooth conjugacy class of circle mappings from a critical commuting pair.

We can metrize the space of $\mathrm{C}^{r}$-smooth commuting pairs considered modulo an affine conjugacy as follows (see $[\mathrm{dFdM1}])$. Let $\zeta_{1}=\left(\eta_{1}, \xi_{1}\right), \zeta_{2}=\left(\eta_{2}, \xi_{2}\right)$ be two such pairs, and denote $w_{i}: \mathbf{C} \rightarrow \mathbf{C}$ a Möbius transformation which maps the ordered triple of points $\eta_{i}(0), 0, \xi_{i}(0)$ to $0,1 / 2,1$. The $\mathrm{C}^{r}$-distance between $\zeta_{1}$ and $\zeta_{2}$ is set to be

$$
\begin{aligned}
\operatorname{dist}_{\mathrm{C}^{r}}\left(\zeta_{1}, \zeta_{2}\right)=\max \{\mid & \xi_{1}(0) / \eta_{1}(0)-\xi_{2}(0) / \eta_{2}(0) \mid \\
& \left.\operatorname{dist}_{\mathrm{C}^{r}}\left(w_{1} \circ \zeta_{1} \circ w_{1}^{-1}, w_{2} \circ \zeta_{2} \circ w_{2}^{-1}\right)\right\} .
\end{aligned}
$$

Let $f$ be a critical circle mapping, whose rotation number $\rho$ has a continued fraction expansion (1.1) with at least $m+1$ terms, and let $p_{m} / q_{m}=\left[r_{0}, \ldots, r_{m-1}\right]$. The pair of iterates $f^{q_{m+1}}$ and $f^{q_{m}}$ restricted to the circle arcs $\mathrm{I}_{m}$ and $\mathrm{I}_{m+1}$ correspondingly can be viewed as a critical commuting pair in the following way. Let $\bar{f}$ be the lift of $f$ to the real line satisfying $\bar{f}^{\prime}(0)=0$, and $0<\bar{f}(0)<1$. For each $m>0$ let $\overline{\mathrm{I}}_{m} \subset \mathbf{R}$ denote the closed interval adjacent to zero which projects down to the interval $\mathrm{I}_{m}$. Let $\tau: \mathbf{R} \rightarrow \mathbf{R}$ denote the translation $x \mapsto x+1$. Let $\eta: \overline{\mathrm{I}}_{m} \rightarrow \mathbf{R}$, $\xi: \overline{\mathbf{I}}_{m+1} \rightarrow \mathbf{R}$ be given by $\eta \equiv \tau^{-p_{m+1}} \circ \bar{f}^{q_{m+1}}, \xi \equiv \tau^{-p_{m}} \circ \bar{f}^{q_{m}}$. Then the pair of maps $\left(\eta\left|\overline{\mathbf{I}}_{m}, \xi\right| \overline{\mathbf{I}}_{m+1}\right)$ forms a critical commuting pair corresponding to $\left(f^{q_{m+1}}\left|\mathbf{I}_{m}, f^{q_{m}}\right| \mathbf{I}_{m+1}\right)$. Henceforth we shall simply denote this commuting pair by

$$
\left(f^{q_{m+1}}\left|\mathbf{I}_{m}, f^{q_{m}}\right| \mathbf{I}_{m+1}\right) .
$$

This allows us to readily identify the dynamics of the above commuting pair with that of the underlying circle map, at the cost of a minor abuse of notation. 
Following $[\mathrm{dFdM1}$, we say that the height $\chi(\zeta)$ of a critical commuting pair $\zeta=(\eta, \xi)$ is equal to $r$, if

$$
0 \in\left[\eta^{r}(\xi(0)), \eta^{r+1}(\xi(0))\right] .
$$

If no such $r$ exists, we set $\chi(\zeta)=\infty$, in this case the map $\eta \mid \mathrm{I}_{\eta}$ has a fixed point. For a pair $\zeta$ with $\chi(\zeta)=r<\infty$ one verifies directly that the mappings $\eta \mid\left[0, \eta^{r}(\xi(0))\right]$ and $\eta^{r} \circ \xi \mid \mathrm{I}_{\xi}$ again form a commuting pair. For a commuting pair $\zeta=(\eta, \xi)$ we will denote by $\widetilde{\zeta}$ the pair $\left(\widetilde{\eta}\left|\widetilde{I_{\eta}}, \widetilde{\xi}\right| \widetilde{I_{\xi}}\right)$ where tilde means rescaling by the linear factor $\lambda=-\frac{1}{\left|I_{\eta}\right|}$.

Definition 2.2. - The renormalization of a real commuting pair $\zeta=(\eta, \xi)$ is the commuting pair

$$
\mathscr{R} \zeta=\left(\widetilde{\eta^{r} \circ \xi}\left|\widetilde{I_{\xi}}, \tilde{\eta}\right|\left[0, \widetilde{\eta^{r}(\xi(0))}\right]\right) .
$$

The non-rescaled pair $\left(\eta^{r} \circ \xi\left|\mathrm{I}_{\xi}, \eta\right|\left[0, \eta^{r}(\xi(0))\right]\right)$ will be referred to as the prerenormalization $p \mathscr{R} \zeta$ of the commuting pair $\zeta=(\eta, \xi)$.

For a pair $\zeta$ we define its rotation number $\rho(\zeta) \in[0,1]$ to be equal to the continued fraction $\left[r_{0}, r_{1}, \ldots\right]$ where $r_{i}=\chi\left(\mathscr{R}^{i} \zeta\right)$. In this definition $1 / \infty$ is understood as 0 , hence a rotation number is rational if and only if only finitely many renormalizations of $\zeta$ are defined; if $\chi(\zeta)=\infty, \rho(\zeta)=0$. Thus defined, the rotation number of a commuting pair can be viewed as a rotation number in the usual sense:

Proposition 2.1. - The rotation number of the mapping $\mathrm{F}_{\zeta}$ is equal to $\rho(\zeta)$.

There is an advantage in defining $\rho(\zeta)$ using a sequence of heights in removing the ambiguity in prescribing a continued fraction expansion to rational rotation numbers in a renormalization-natural way.

For $\rho=\left[r_{0}, r_{1}, \ldots\right] \in[0,1]$ let us set

$$
\mathrm{G}(\rho)=\left[r_{1}, r_{2}, \ldots\right]=\left\{\frac{1}{\rho}\right\},
$$

where $\{x\}$ denotes the fractional part of a real number $x$ ( $G$ is usually referred to as the Gauss map). As follows from the definition,

$$
\rho(\mathscr{R} \zeta)=\mathrm{G}(\rho(\zeta))
$$

for a real commuting pair $\zeta$ with $\rho(\zeta) \neq 0$.

The renormalization of the real commuting pair (2.1), associated to some critical circle map $f$, is the rescaled pair $\left(\widetilde{f^{q_{m+2}} \mid \widetilde{\mathrm{I}_{m+1}}}, \widetilde{f^{q_{m+1}}} \mid \widetilde{\mathrm{I}_{m+2}}\right)$. Thus for a given critical circle map $f$ the renormalization operator recovers the (rescaled) sequence of the first return maps:

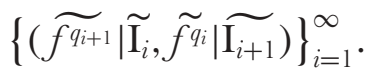




\subsection{Explanation of universality: Lanford's Program}

The golden-mean universality phenomenon described above was explained in [ORSS] and [FKS] by a conjectural hyperbolicity property of the operator $\mathscr{R}$. The conjecture was later generalized by various authors, particularly by Lanford [Lan2], to account for more complex universalities. We present it below in its most general form, known as Lanford's Program.

First, a definition. A critical commuting pair is a commuting pair $(\eta, \xi)$ whose maps are real-analytic. We shall also impose a technical assumption that $\xi$ analytically extends to an interval $(a, b) \ni 0$ with $\xi(a, b) \supset[\eta(0), \xi(0)]$, and has a single critical point 0 in this interval. The space of critical commuting pairs modulo affine conjugacy will be denoted by $\mathfrak{C}$; its subset consisting of pairs $\zeta$ with $\chi(\zeta)=\infty$ will be denoted by $\mathfrak{S}_{\infty}$. Renormalization is a transformation $\mathscr{R}: \mathfrak{C} \backslash \mathfrak{C}_{\infty} \rightarrow \mathfrak{C}$. It is not difficult to show (see [Ya2]) that this transformation is injective:

Proposition 2.2. - The map $\mathscr{R}: \mathfrak{C} \backslash \mathfrak{S}_{\infty} \rightarrow \mathfrak{C}$ is one-to-one.

To outline Lanford's Program, let us give an informal description of the action of renormalization on the space of critical commuting pairs.

Let $\rho=\phi(\theta): \mathbf{T} \rightarrow \mathbf{T}$ be a monotone continuous function such that $\phi^{-1}(\rho)$ is a point for $\rho$ irrational and an interval otherwise. An example of such a function is the dependence $\theta \mapsto \rho\left(f_{\theta}\right)$ of the rotation number of a standard map on the parameter. Imagine the space of critical commuting pairs as an infinite-dimensional cylinder $\mathfrak{C}=\mathbf{T} \times \mathfrak{C}^{\prime}$, where the rotation number of a commuting pair $\zeta(\theta, \cdot)$ with the equatorial coordinate $\theta \in \mathbf{T}$ is $\rho(\theta)$. The cylinder $\mathfrak{C}$ is partitioned into strips (cf. Figure 2)

$$
\mathfrak{S}_{r}=\left\{\zeta \in \mathfrak{C} \mid \rho(\zeta)=\left[r, r_{1}, \ldots\right]\right\} \text { for } r=1, \ldots, \infty .
$$

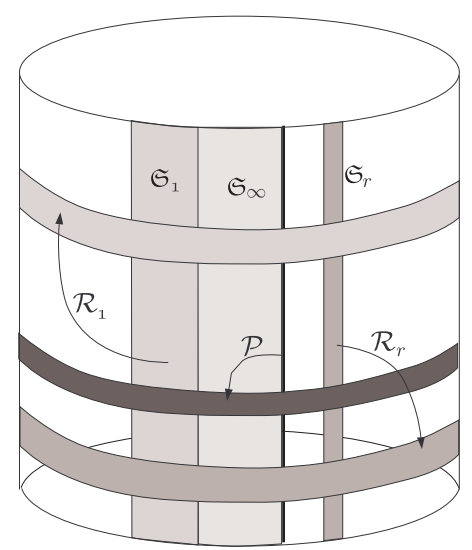

FIG. 2. 
A boundary component of the strip $\mathfrak{S}_{\infty}$ is the hypersurface $\mathfrak{P}_{\infty} \subset \mathfrak{S}_{\infty}$ with the property that a pair $\zeta \in \mathfrak{P}_{\infty}$ if and only if it has a single fixed point with unit eigenvalue ( $\zeta$ is a "parabolic pair"). The sets $\mathfrak{S}_{r}$ accumulate on $\mathfrak{P}_{\infty}$ in a clockwise direction.

It is natural to think of the transformation $\mathscr{R}: \mathfrak{C} \backslash \mathfrak{S}_{\infty} \rightarrow \mathfrak{C}$ as being defined piecewise, given on each $\mathfrak{S}_{r}, r \neq \infty$ by the formula:

$$
\mathscr{R}_{r}:(\eta, \xi) \mapsto\left(\eta, \eta^{r} \circ \xi\right) .
$$

The operator $\mathscr{R}_{r}$ expands the strip $\mathfrak{S}_{r}$ in the equatorial direction, mapping it onto a thin equatorial cylinder, which intersects all the vertical strips. We may close the domain of $\mathscr{R}$ by adjoining the hypersurface $\mathfrak{P}_{\infty}$ to it and setting $\mathscr{R}$ on $\mathfrak{P}_{\infty}$ to an appropriately defined (multivalued) parabolic renormalization transformation $\mathscr{P}$, with this convention the image of $\mathfrak{P}_{\infty}$ is also an equatorial cylinder. Lanford conjectured that this picture can be appropriately endowed by a smooth metric, so that the operator $\mathscr{R}$ is uniformly expanding in the $\theta$ direction and uniformly contracting in the complementary directions. Thus $\mathscr{R}$ posesses an infinite-dimensional "horseshoe" structure. The "boxes"

$$
\begin{aligned}
\mathscr{R}_{r_{-1}}\left(\mathfrak{S}_{r_{-1}}\right. & \cap \mathscr{R}_{r_{-2}}\left(\mathfrak{S}_{r_{-2}} \cap \cdots\left(\mathscr{R}_{r_{-n}} \mathfrak{S}_{r_{-n}}\right) \cdots\right) \\
& \cap \mathfrak{S}_{r_{0}} \cap \mathscr{R}_{r_{0}}^{-1}\left(\mathscr{R}_{r_{1}}^{-1}\left(\cdots \mathscr{R}_{r_{n-1}}^{-1}\left(\mathfrak{S}_{r_{n}}\right) \cdots\right)\right.
\end{aligned}
$$

are exponentially small in $n$, and their intersection is the $\mathscr{R}$-invariant hyperbolic Cantor set $\mathscr{A}$ parametrized by the bi-infinite sequences of $r_{i} \in \mathbf{N} \cup\{\infty\}$. The set $\mathscr{A}$ is a "horseshoe" attractor for the renormalization operator,

$$
\operatorname{dist}\left(\mathscr{R}^{n} \zeta, \mathscr{A}\right) \rightarrow 0
$$

at a geometric rate. The action of $\mathscr{R}$ on $\mathscr{A}$ is conjugate to the two-sided shift.

Let us conclude this exposition by formalizing the Hyperbolicity Conjecture as follows:

Renormalization Hyperbolicity Conjecture (Lanford's Program). - The renormalization operator $\mathscr{R}$ in the space of critical commuting pairs possesses a "horseshoe" attractor $\mathscr{A}$ on which its action is conjugated to the two-sided shift. Moreover, there exists a renormalizationinvariant space of critical commuting pairs with the structure of an infinite dimensional smooth manifold, with respect to which $\mathscr{A}$ is a hyperbolic set with one-dimensional expanding direction. If $t$ denotes the expanded coordinate in a local chart, then the dependence $t \mapsto \rho(t, \cdot)$ is continuous and (not strictly) monotone.

It is not difficult to see that this Conjecture explains, in particular, the golden mean universality phenomenon we described. Indeed, it implies the existence of a unique fixed point $\zeta_{*}=\left(\eta_{*}, \xi_{*}\right) \in \mathscr{A}$ with $\rho\left(\zeta_{*}\right)=(\sqrt{5}-1) / 2$. The fixed point $\zeta_{*}$ is hyperbolic, let us denote its unique expanding eigenvalue $\delta_{*}$ and set the 
scaling factor $\left|\mathrm{I}_{\xi_{*}}\right| /\left|\mathrm{I}_{\eta_{*}}\right|=\lambda_{*}$. Then for any $\zeta$ with $\rho(\zeta)=(\sqrt{5}-1) / 2$ we have $\mathscr{R}_{1}^{\circ} \zeta \rightarrow \zeta_{*}$ which explains the universality of $\lambda_{*}$. Moreover, let $\zeta_{\mu}$ be a monotone one-parameter family of critical commuting pairs. Since the slices $\left(\mathscr{R}_{1}\right)^{-n}\left(\mathfrak{S}_{1}\right)$ accumulate at the local stable manifold of $\zeta_{*}$ at the geometric rate $1 / \delta_{*}$, they have "width" $\sim \delta_{*}^{-n}$. Provided the family $\zeta_{\mu}$ crosses the local stable manifold of $\zeta_{*}$ transversely, this implies that the sizes of parameter intervals $\mathscr{I}_{n}=\left\{\mu: \zeta_{\mu} \in \mathfrak{S}_{n}\right\}$ decay at the rate $1 / \delta_{*}$ as well.

\section{The passage from smooth to holomorphic}

The golden-mean universality is observed in any one-parameter family of smooth critical circle maps. We chose to formulate the Hyperbolicity Conjecture for analytic commuting pairs, in part to ensure that the action of renormalization is injective. A deeper reason is the following. In 1986 Eckmann and Epstein [EE] introduced a space of critical commuting pairs now known as the Epstein class. They showed that this class is invariant under the action of $\mathscr{R}$, and constructed the golden-mean fixed point of $\mathscr{R}$ in this class using the methods of geometric complex analysis. It was further shown by various people, such as Sullivan (in the unimodal case), Świastek, Herman, and Yoccoz, that the renormalizations of any $\mathrm{C}^{3}$-smooth commuting pair with an irrational rotation number converge to the Epstein class, at a geometric rate in the $\mathrm{C}^{2}$-metric. Below, after some preliminaries, we define the Epstein class, and formulate these results more precisely.

\subsection{Carathéodory topology on a space of branched coverings}

Consider the collection $\mathfrak{X}$ of all triplets $(\mathrm{U}, u, f)$, where $\mathrm{U} \subset \mathbf{C}$ is a topological disk different from the whole plane, $u \in \mathrm{U}$, and $f: \mathbf{U} \rightarrow \mathbf{G}$ is a three-fold analytic branched covering map, with the only branch point at $u$. We will topologize $\mathfrak{X}$ as follows (cf. [McMl]).

Let $\left\{\left(\mathrm{U}_{n}, u_{n}\right)\right\}$ be a sequence of open connected regions $\mathrm{U}_{n} \subset \mathbf{C}$ with marked points $u_{n} \in \mathrm{U}_{n}$. Recall that this sequence Carathéodory converges to a marked region $(\mathrm{U}, u)$ if:

- $u_{n} \rightarrow u \in \mathrm{U}$, and

- for any Hausdorff limit point $\mathrm{K}$ of the sequence $\hat{\mathbf{C}} \backslash \mathrm{U}_{n}, \mathrm{U}$ is a component of $\hat{\mathbf{C}} \backslash \mathrm{K}$.

For a simply connected $\mathrm{U} \subset \mathbf{G}$ and $u \in \mathrm{U}$ let $\mathrm{R}_{(\mathrm{U}, u)}: \mathbf{D} \rightarrow \mathrm{U}$ denote the inverse Riemann mapping with normalization $\mathrm{R}_{(\mathrm{U}, u)}(0)=u, \mathrm{R}_{(\mathrm{U}, u)}^{\prime}(0)>0$. By a classical result of Carathéodory, the Carathédory convergence of simply-connected regions 
$\left(\mathrm{U}_{n}, u_{n}\right) \rightarrow(\mathrm{U}, u)$ is equivalent to the locally uniform convergence of the inverse Riemann mappings $\mathrm{R}_{\left(\mathrm{U}_{n}, u_{n}\right)}$ to $\mathrm{R}_{(\mathrm{U}, u)}$.

For positive numbers $\epsilon_{1}, \epsilon_{2}, \epsilon_{3}$ and compact subsets $\mathrm{K}_{1}$ and $\mathrm{K}_{2}$ of the open unit disk $\mathbf{D}$, let the neighborhood $\mathscr{U}_{\epsilon_{1}, \epsilon_{2}, \epsilon_{3}, \mathrm{~K}_{1}, \mathrm{~K}_{2}}(\mathrm{U}, u, f)$ of an element $(\mathrm{U}, u, f) \in \mathfrak{X}$ be the set of all $(\mathrm{V}, v, g) \in \mathfrak{X}$, for which:

- $|u-v|<\epsilon_{1}$,

- $\sup _{z \in \mathrm{K}_{1}}\left|\mathrm{R}_{(\mathrm{V}, v)}(z)-\mathrm{R}_{(\mathrm{U}, u)}(z)\right|<\epsilon_{2}$,

- and $\mathrm{R}_{(\mathrm{U}, u)}\left(\mathrm{K}_{2}\right) \subset \mathrm{V}$, and $\sup _{z \in \mathrm{R}_{(\mathrm{U}, u)}\left(\mathrm{K}_{2}\right)}|f(z)-g(z)|<\epsilon_{3}$.

One verifies that the sets $\mathscr{U}_{\epsilon_{1}, \epsilon_{2}, \epsilon_{3}, \mathrm{~K}_{1}, \mathrm{~K}_{2}}(\mathrm{U}, u, f)$ form a base of a topology on $\mathfrak{X}$, which we will call Carathéodory topology. This topology is clearly Hausdorff, and the convergence of a sequence $\left(\mathrm{U}_{n}, u_{n}, f_{n}\right)$ to $(\mathrm{U}, u, f)$ is equivalent to the Carathéodory convergence of the marked regions $\left(\mathrm{U}_{n}, u_{n}\right) \rightarrow(\mathrm{U}, u)$ as well as a locally uniform convergence $f_{n} \rightarrow f$.

\subsection{The Epstein class}

An orientation preserving interval homeomorphism $g: \mathrm{I}=[0, a] \rightarrow g(\mathrm{I})=\mathrm{J}$ belongs to the Epstein class $\mathscr{E}$ if it extends to an analytic three-fold branched covering map of a topological disk $\mathrm{G} \supset \mathrm{I}$ onto the double-slit plane $\mathbf{C}_{\tilde{J}}$, where $\tilde{\mathrm{J}} \supset \mathrm{clJ}$. Any map $g$ in the Epstein class can be decomposed as

$$
g=Q_{c} \circ h,
$$

where $\mathrm{Q}_{c}(z)=z^{3}+c$, and $h: \mathrm{I} \rightarrow[0, b]$ is a univalent map $h: \mathrm{G} \rightarrow \Delta(h)$ onto the complex plane with six slits, which triple covers $\mathbf{C}_{\tilde{J}}$ under the cubic map $\mathrm{Q}_{\mathfrak{c}}(z)$.

For any $s \in(0,1)$, let us introduce a smaller class $\mathscr{E}_{s} \subset \mathscr{E}$ of Epstein mappings $g: \mathrm{I}=[0, a] \rightarrow \mathrm{J} \subset \tilde{\mathrm{J}}$ for which both $|\mathrm{I}|$ and $\operatorname{dist}(\mathrm{I}, \mathrm{J})$ are $s^{-1}$-commensurable with $|\mathrm{J}|$, the length of each component of $\tilde{\mathrm{J}} \backslash \mathrm{J}$ is at least $s|\mathrm{~J}|$, and $g^{\prime}(a)>s$. We will often refer to the space $\mathscr{E}$ as the Epstein class, and to each $\mathscr{E}_{S}$ as an Epstein class.

We say that a commuting pair $(\eta, \xi) \in \mathfrak{C}$ belongs to the (an) Epstein class if both of its maps do. Similarly, a critical circle map $f$ is Epstein if $\mathscr{R} f$ is in the Epstein class. It immediately follows from the definitions that:

Lemma 3.1. - If a renormalizable commuting pair $\zeta$ is in the Epstein class, then the same is true for $\mathscr{R} \zeta$.

Let us make a note of an important compactness property of $\mathscr{E}_{s}$ 
Lemma 3.2 (Lemma 2.10 [Ya2]). - Let $s \in(0,1)$. The collection of normalized maps $g \in \mathscr{E}_{s}$ with $\mathrm{I}=[0,1]$, with marked domains $(\mathrm{U}, 0)$ is sequentially compact with respect to Carathéodory topology.

\subsection{Real a priori bounds}

An excellent exposition of the results of Herman, Świastek, and Yoccoz on the real geometry of critical circle maps is found in [dFdM1]. We formulate some of the main results.

Real a priori bounds of Herman and Siriatek. - There exists a universal constant K such that the following holds. Let $f$ be a critical circle map. Denote $\Pi_{m}$ the partition of the circle by the iterates

$$
\left\{f^{q_{m} i}(0)\right\}_{i=0}^{q_{m-1}-1} \cup\left\{f^{q_{m-1} i}(0)\right\}_{i=0}^{q_{m}-1} \cup\left\{f^{q_{m-1}+q_{m} i}(0)\right\}_{i=0}^{q_{m-1}-1} \cup\left\{f^{q_{m}+q_{m-1} i}(0)\right\}_{i=0}^{q_{m}-1}
$$

(note that $\mathrm{I}_{m}$ and $\mathrm{I}_{m-1}$ are two adjacent intervals of the partition). Then there exists $\mathrm{M}=$ $\mathrm{M}(f)$ such that for every $m>\mathrm{M}$ any two adjacent intervals of the partition are $\mathrm{K}$ commensurable. Moreover, the $\mathrm{G}^{1}$-norm of the renormalizations $\left\{\mathscr{R}^{m}(f)\right\}_{m=\mathrm{M}}^{\infty}$ is bounded by $\mathrm{K}$.

Utilizing the above bounds Herman has shown in [He] (see [dFdM1] for a published account):

Theorem 3.3. - Any two critical circle maps with the same irrational rotation numbers are quasisymmetrically conjugate.

The importance of the Epstein class lies in the fact that all $\mathrm{C}^{1}$-limit points of the sequence $\left\{\mathscr{R}^{m}(f)\right\}_{m=\mathrm{M}}^{\infty}$ are in $\mathscr{E}_{s}$ for a universal value of $s>0$. A more precise formulation of this was proved in the recent work of de Faria and de Melo $[\mathrm{dFdM1}]$ :

Lemma 3.4. - There exists a universal constant $s>0$ such that the following holds. Let $f \in \mathrm{C}^{r}, \quad(r \geq 3)$ be a critical circle map with an irrational rotation number. Then the sequence of real commuting pairs $\mathscr{R}^{m}(f)=\left(\widetilde{f^{q_{m+1}} \mid}\left|\widetilde{\mathrm{I}_{m}}, \widetilde{f^{q_{m}}}\right| \widetilde{\mathrm{I}_{m+1}}\right)$ is bounded in $\mathrm{C}^{r-1}$-metric, and $\mathrm{C}^{r-1}$-converges to $\mathscr{E}_{s}$ at a geometric rate.

In particular, for a critical circle map $f \in \mathscr{E}$ there exists $\sigma>0$ such that all its renormalizations are contained in $\mathscr{E}_{\sigma}$. Moreover, the constant $\sigma$ can be chosen independent on $f$, after skipping the first few renormalizations.

Finally, let us formulate a technical statement about Epstein pairs to be used further in the paper: 
Lemma 3.5 (Lemma 2.13, [Ya2]). - Let $\zeta=(\eta, \xi) \in \mathscr{E}$ be a critical commuting pair with $\rho(\zeta)=0$, which appears as a limit of a sequence $\left\{\zeta_{n}\right\} \subset \mathscr{E}$ with $\rho\left(\zeta_{n}\right) \in \mathbf{R} \backslash \mathbf{Q}$. Then the map $\eta$ has a unique fixed point in the interval $\mathrm{I}_{\eta}$, which is necessarily parabolic, with multiplier one.

A commuting pair $\zeta=(\eta, \xi) \in \mathscr{E}$ will be called parabolic if the map $\eta$ has a unique fixed point in $\mathrm{I}_{\eta}$, which has a unit multiplier; this point will usually be denoted $p_{\eta}$. Note, that by virtue of its uniqueness, $p_{\eta}$ has to be globally attracting on one side for the interval homeomorphism $\left.\eta\right|_{I_{\eta}}$, it is globally attracting on the other side under $\eta^{-1}$.

\section{Outline of the results}

\subsection{Known results}

The early efforts to prove the Renormalization Hyperbolicity Conjecture culminated in an argument of Mestel [Mes], who established the existence and local hyperbolicity properties of a fixed point of $\mathscr{R}_{1}$ in $\mathrm{C}^{\infty}$. The argument was based on rigorous computer estimates. This approach was developed by Lanford in the context of the renormalization of unimodal maps. One of its major drawbacks is its local nature; neither the uniqueness of the fixed point, nor global hyperbolicity of $\mathscr{R}_{1}$ are established.

In his address to ICM-86 in Berkeley Sullivan [Sull] outlined a program of constructing the invariant set of renormalization of unimodal maps and its stable set by means of the Teichmüller theory, which he subsequently carried out (see [Sul2], [MvS]). Sullivan's program has been adapted to the setting of critical circle mappings by de Faria [dF1],[dF2]. De Faria has defined, in particular, extensions of Epstein commuting pairs to holomorphic dynamical systems in the plane, which are analogous to quadratic-like mappings in the unimodal renormalization theory. We discuss these extensions, called holomorphic commuting pairs, in §5. Using Sullivan's Riemann surface laminations machinery, de Faria constructed a horseshoe attractor $\mathscr{A}_{\mathrm{B}}$ for the renormalization operator acting on the space of Epstein commuting pairs of type bounded by some constant B (this corresponds to restricting the operator $\mathscr{R}$ to the finite collection of vertical strips $\cup \mathfrak{S}_{r}, r \leq \mathrm{B}$ in Figure 2). In a recent paper [Ya2] we generalized the results of de Faria to Epstein commuting pairs of unbounded combinatorial type. Denote $\bar{\Sigma}$ the space of bi-infinite sequences $\left(\ldots, r_{-k}, \ldots, r_{-1}, r_{0}, r_{1}, \ldots, r_{k}, \ldots\right)$ with $r_{i} \in \mathbf{N} \cup\{\infty\}$ equipped with the weak topology. Our theorem completely settles the first part of Lanford's Program:

Theorem 4.1 (Renormalization horseshoe). - There exists an $\mathscr{R}$-invariant closed set $\mathscr{A} \subset \mathscr{E}$ consisting of pairs with irrational rotation numbers and parabolic pairs with the 
following properties. The action of $\mathscr{R}$ on $\mathscr{A}$ is topologically conjugate to the two-sided shift $\sigma: \bar{\Sigma} \rightarrow \bar{\Sigma}:$

$$
i \circ \mathscr{R} \circ i^{-1}=\sigma
$$

and if $\zeta=i^{-1}\left(\ldots, r_{-k}, \ldots, r_{-1}, r_{0}, r_{1}, \ldots, r_{k}, \ldots\right)$ then $\rho(\zeta)=\left[r_{0}, r_{1}, \ldots, r_{k}, \ldots\right]$. For any $\zeta \in \mathscr{E}$ with irrational rotation number we have

$$
\mathscr{R}^{n} \zeta \rightarrow \mathscr{A}
$$

in the Carathéodory topology. Moreover, for any two pairs $\zeta, \zeta^{\prime} \in \mathscr{E}$ with $\rho(\zeta)=\rho\left(\zeta^{\prime}\right)$ we have

$$
\operatorname{dist}\left(\mathscr{R}^{n} \zeta, \mathscr{R}^{n} \zeta^{\prime}\right) \rightarrow 0
$$

for the uniform distance between analytic extensions of the renormalized pairs on compact sets.

The proof of Theorem 4.1 combines the ideas of McMullen from [McM2] with a priori bounds obtained in [Yal]. We consider the geometric limits of the rescaled sequences of renormalizations

$$
\zeta, \mathscr{R} \zeta, \ldots, \mathscr{R}^{n} \zeta
$$

These limits which we call bi-infinite limiting renormalization towers (see $\S 5$ for the definition) should be thought of as bi-infinite sequences of nested holomorphic commuting pairs in the plane. Each tower corresponds to a single point in the attractor $\mathscr{A}$, and the result follows from a Rigidity Theorem [Ya2]:

Theorem 4.2 (Tower rigidity). — Any two towers with the same rotation numbers are affinely conjugated.

As far as the hyperbolicity aspects of Lanford's Program, the following is known. In the case of a bounded type rotation number, de Faria and de Melo [dFdM2] strengthened the original result of de Faria, utilizing a technique of McMullen [McM2] to show that the rate of converegence to $\mathscr{A}_{\mathrm{B}}$ is geometric:

Theorem 4.3 ([dFdM2]). - For every $\mathrm{B} \in \mathbf{N}$ and every $r \in \mathbf{N} \cup 0$ there exists $\alpha_{r, \mathrm{~B}}<1$ such that for every $\zeta \in \mathscr{E}$ with $\rho(\zeta) \in \mathbf{R} \backslash \mathbf{Q}$ of type bounded by $\mathrm{B}$ and for every $\hat{\zeta} \in \mathscr{A}_{\mathrm{B}}$ with $\rho(\hat{\zeta})=\rho(\zeta)$ we have

$$
\operatorname{dist}_{\mathrm{C}^{r}}\left(\mathscr{R}^{n} \zeta, \mathscr{R}^{n} \hat{\zeta}\right)<\text { const } \cdot\left(\alpha_{r, \mathrm{~B}}\right)^{n} .
$$

Despite the difficulty in imposing a manifold structure on the space of commuting pairs compatible with the analyticity of $\mathscr{R}$, it has long been known how one may construct an unstable direction in such a manifold. A version of this construction was first shown to us by Folkert Tangerman, who participated in its discussion in Sullivan's seminar at the Graduate Center of CUNY. We give a rather simple argument in $\S 9$. 


\subsection{Statement of the main result}

First, some definitions. Suppose, B is a complex Banach space whose elements are functions of the complex variable. Let us say that the real slice of $\mathrm{B}$ is the real Banach space $\mathrm{B}^{\mathbf{R}}$ consisting of the real-symmetric elements of $\mathrm{B}$. If $\mathrm{X}$ is a Banach manifold modelled on $\mathrm{B}$ with the atlas $\left\{\Psi_{\gamma}\right\}$ we shall say that $\mathrm{X}$ is real-symmetric if $\Psi_{\gamma_{1}} \circ \Psi_{\gamma_{2}}^{-1}\left(\mathrm{~B}^{\mathbf{R}}\right) \subset \mathrm{B}^{\mathbf{R}}$ for any pair of indices $\gamma_{1}, \gamma_{2}$. The real slice of $\mathrm{X}$ is then defined as the real Banach manifold $\mathrm{X}^{\mathbf{R}} \subset \mathrm{X}$ given by $\Psi_{\gamma}^{-1}\left(\mathrm{~B}^{\mathbf{R}}\right)$ in a local chart $\Psi_{\gamma}$. An operator A defined on a subset of $\mathrm{X}$ is real-symmetric if $\mathrm{A}\left(\mathrm{X}^{\mathbf{R}}\right) \subset \mathrm{X}^{\mathbf{R}}$. A particular example we shall introduce in $\S 7$ is a real-analytic manifold $\mathfrak{C}_{\mathrm{U}}$ of critical cylinder maps. Its real slice $\mathfrak{M} \equiv \mathfrak{C}_{U}^{\mathbf{R}}$ consists of critical circle maps defined in an annulus $\mathrm{U} \supset \mathbf{T}$ in the cylinder $\mathbf{C} / \mathbf{Z}$.

We will construct a closed equivalence relation denoted $\underset{\text { conf }}{\sim}$ between commuting pairs in the Epstein class $\mathscr{E}_{s}$ (with $s$ being as in Lemma 3.4) with the property that if $\zeta_{1} \underset{\text { conf }}{\sim} \zeta_{2}$, then the analytic extensions of the commuting pairs are conjugate in a specific neighborhood of the interval of definition by a conformal change of coordinates. In particular, $\rho\left(\zeta_{1}\right)=\rho\left(\zeta_{2}\right)$. Moreover, there exists a value $\mathrm{N} \in \mathbf{N}$ such that if $\zeta_{1}$ and $\zeta_{2}$ are N-times renormalizable, then $\mathscr{R}^{\mathrm{N}} \zeta_{1} \underset{\text { conf }}{\mathscr{R}^{\mathrm{N}} \zeta_{2}}$. This last property implies that periodic orbits of $\mathscr{R}^{\mathrm{N}}$ constructed in Theorem 4.1 project to the quotient space.

The main result of this paper is the Renormalization Hyperbolicity Theorem in the following form:

Theorem 4.4 (Renormalization Hyperbolicity). - There exists a real-symmetric analytic operator $\mathscr{R}_{\text {cyl }}$ from an open subset of $\mathfrak{C}_{\mathrm{U}}$ to $\mathfrak{C}_{\mathrm{U}}$ (thus $\mathscr{R}_{\text {cyl }}$ maps an open subset of $\mathfrak{M}$ into $\mathfrak{M})$, such that the following holds. For every $r \neq \infty$ there is a canonical homeomorphism onto the image

$$
\mathfrak{S}_{r} \cap \mathscr{E}_{s} / \underset{\text { conf }}{\sim} \stackrel{\iota_{r}}{\longrightarrow} \mathfrak{M}
$$

such that $\rho\left(\iota_{r}(\zeta)\right)=\rho(\mathscr{R}(\zeta))$, and $\iota_{r} \circ \mathscr{R}^{\mathrm{N}} \circ \iota_{r}^{-1} \equiv \mathscr{R}_{\mathrm{cyl}}$. For every periodic point $p \in \mathfrak{S}_{r}$ of the operator $\mathscr{R}^{\mathrm{N}}$ there exists $\ell \in \mathbf{N}$ such that the point $\iota_{r}(p)$ is a hyperbolic fixed point of $\left.\mathscr{R}_{\mathrm{cyl}}^{\ell}\right|_{\mathfrak{M}}$, with a one-dimensional unstable direction.

Note, in particular, that we have found a way to define a renormalization for critical circle maps themselves, without recourse to critical commuting pairs. Our theorem implies the existence of universal constants associated to every rotation number of a periodic type, and in particular we get a version of the golden-mean universality:

Corollary 4.5 (Golden-Mean Universality). - There exists a pair of universal constants $\delta>1$ and $\lambda>0$, such that the following holds. Let $\left\{f_{\mu}\right\}$ be a one-parameter family of analytic 
critical circle maps with a $\mathrm{C}^{1}$-smooth dependence on the parameter $\mu$. Denote $\tilde{f}_{\mu}: \mathbf{R} \rightarrow \mathbf{R}$ a continuously chosen lift of $f_{\mu}: \mathbf{T} \rightarrow \mathbf{T}$, and assume additionally that $\partial \tilde{f}_{\mu}(x) / \partial \mu>0$ for any $x \in \mathbf{R}$ (the Arnold's family is one example of such family). Suppose $\rho\left(f_{\mu_{*}}\right)=(\sqrt{5}-1) / 2$ and denote $\mathrm{I}_{n}$ the closed interval of values of the parameter for which $\rho\left(f_{\mu}\right)=p_{n} / q_{n}$ where $p_{n} / q_{n}$ is the $n$-th convergent of the golden mean. Then:

- $\left|\mathrm{I}_{k+\mathrm{M} M}\right| / \delta^{\mathrm{M}} \rightarrow a>0$ for some $\mathrm{M} \in \mathbf{N}$;

- $\left|f_{\mu_{*}}^{q_{n}}(0)\right| /\left|f_{\mu_{*}}^{q_{n+1}}(0)\right| \rightarrow \lambda$ geometrically fast.

We remark that the second statement already follows from [dFdM2].

\section{Holomorphic commuting pairs}

\subsection{Definitions}

De Faria [dF1,dF2] introduced holomorphic commuting pairs to apply Sullivan's Riemann surface laminations technique to the renormalization of critical circle maps. They are suitably defined holomorphic extensions of critical commuting pairs which replace Douady-Hubbard polynomial-like maps [DH2]. A critical commuting pair $\zeta=\left(\left.\eta\right|_{I_{\eta}},\left.\xi\right|_{I_{\xi}}\right)$ extends to a holomorphic commuting pair $\mathscr{H}$ if there exist four simply-connected $\mathbf{R}$-symmetric domains $\Delta, \mathrm{D}, \mathrm{U}, \mathrm{V}$ such that

- $\overline{\mathrm{D}}, \overline{\mathrm{U}}, \overline{\mathrm{V}} \subset \Delta, \overline{\mathrm{U}} \cap \overline{\mathrm{V}}=\{0\}$; the sets $\mathrm{U} \backslash \mathrm{D}, \mathrm{V} \backslash \mathrm{D}, \mathrm{D} \backslash \mathrm{U}$, and $\mathrm{D} \backslash \mathrm{V}$ are nonempty, connected, and simply-connected, $\mathrm{U} \cap \mathbf{R}=\mathrm{I}_{\eta}, \mathrm{V} \cap \mathbf{R}=\mathrm{I}_{\xi}$;

- mappings $\eta: \mathrm{U} \rightarrow(\Delta \backslash \mathbf{R}) \cup \eta\left(\mathrm{I}_{\eta}\right)$ and $\xi: \mathrm{V} \rightarrow(\Delta \backslash \mathbf{R}) \cup \xi\left(\mathrm{I}_{\xi}\right)$ are onto and univalent;

- $v \equiv \eta \circ \xi: \mathrm{D} \rightarrow(\Delta \backslash \mathbf{R}) \cup v\left(\mathrm{I}_{\mathrm{D}}\right)$ is a three-fold branched covering with a unique critical point at zero, where $\mathrm{I}_{\mathrm{D}}=\mathrm{D} \cap \mathbf{R}$.

We shall call $\zeta$ the commuting pair underlying $\mathscr{H}$, and write $\zeta \equiv \zeta_{\mathscr{C}}$. The domain $\mathrm{D} \cup \mathrm{U} \cup \mathrm{V}$ of a holomorphic commuting pair $\mathscr{H}$ will be denoted $\Omega$ or $\Omega_{\mathscr{H}}$, the

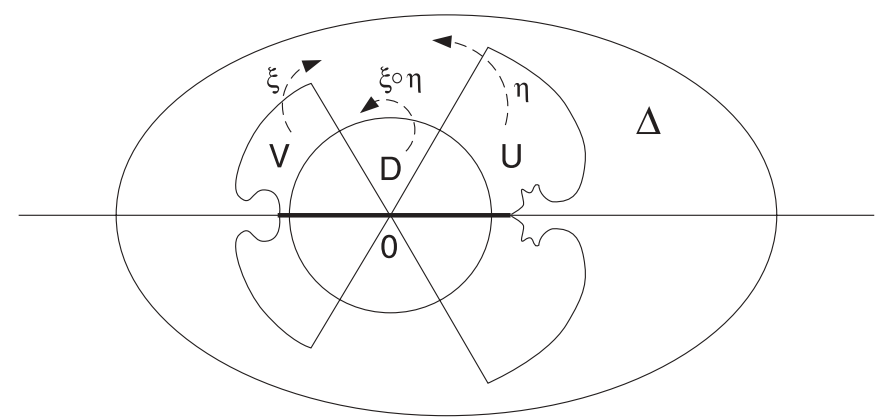

FIG. 3. - A holomorphic commuting pair 
range will be denoted $\Delta$ or $\Delta_{\mathscr{H}}$. The closure of the set of points whose orbit under $\mathscr{H}$ is contained in $\Omega$ will be referred to as the filled Fulia set of $\mathscr{H}$, denoted $\mathrm{K}(\mathscr{H})$. The Fulia set of $\mathscr{H}$ is defined as $\mathrm{J}(\mathscr{H})=\partial \mathrm{K}(\mathscr{H})$.

It is easy to see directly from the definition (cf. [dF2]) that:

Proposition 5.1. - Let $\zeta$ be a commuting pair with $\chi(\zeta)<\infty$. Suppose $\zeta$ is a restriction of a holomorphic commuting pair $\mathscr{H}$, that is $\zeta=\zeta_{\mathscr{H}}$. Then there exists a holomorphic commuting pair $\mathscr{G}$ with range $\Delta_{\mathscr{H}}$, such that $\zeta_{\mathscr{G}}=\mathscr{R} \zeta$.

The shadow of the holomorphic commuting pair $\mathscr{H}$ is the following piecewisedefined holomorphic dynamical system:

$$
\mathrm{S}_{\mathscr{H}}(z)=\left\{\begin{array}{l}
\eta(z), z \in \mathrm{U} \\
\xi(z), z \in \mathrm{V} \\
\xi \circ \eta(z), z \in \mathrm{D} \backslash(\mathrm{U} \cup \mathrm{V}) .
\end{array}\right.
$$

As the next proposition shows one may think of the shadow of a holomorphic commuting pair as an analogue of a cubic-like map:

Proposition 5.2 (Prop. II.4. [dF2]). — Given a holomorphic commuting pair $\mathscr{H}$ as above, consider its shadow $\mathrm{S}_{\mathscr{H}}$. Let $\mathrm{I}=\Omega \cap \mathbf{R}$, and $\mathrm{X}=\mathrm{I} \cup \mathrm{S}_{\mathscr{H}}^{-1}(\mathrm{I})$. Then:

- The restriction of $\mathrm{S}_{\mathscr{H}}$ to $\Omega \backslash \mathrm{X}$ is a regular three fold covering onto $\Delta \backslash \mathbf{R}$.

- $\mathrm{S}_{\mathscr{H}}$ and $\mathscr{H}$ share the same orbits as sets.

We will say that two holomorphic commuting pairs $\mathscr{H}: \Omega_{\mathscr{H}} \rightarrow \Delta_{\mathscr{H}}$ and $\mathscr{G}: \Omega_{\mathscr{G}} \rightarrow \Delta_{\mathscr{G}}$ are conjugate if there is a homeomorphism $h: \Delta_{\mathscr{G}} \rightarrow \Delta_{\mathscr{H}}$ such that

$$
\mathrm{S}_{\mathscr{G}}=h^{-1} \circ \mathrm{S}_{\mathscr{H}} \circ h
$$

In this case we will simply write $\mathscr{G}=h^{-1} \circ \mathscr{H} \circ h$.

\subsection{Complex a priori bounds}

We shall denote by $\mathfrak{H}$ the space of holomorphic commuting pairs $\mathscr{H}: \Omega \rightarrow \Delta$ whose underlying real commuting pair $(\eta, \xi)$ is in the Epstein class. In this case both maps $\eta$ and $\xi$ extend to triple branched coverings $\hat{\eta}: \hat{\mathrm{U}} \rightarrow \Delta \cap \mathbf{C}_{\eta\left(\mathrm{J}_{\eta}\right)}$ and $\hat{\xi}: \hat{V} \rightarrow \Delta \cap \mathbf{C}_{\xi\left(J_{\xi}\right)}$ respectively. We will turn $\mathfrak{H}$ into a topological space by identifying it with a subset of $\mathfrak{X} \times \mathfrak{X}$ by $\mathscr{H} \mapsto(\hat{\mathrm{U}}, 0, \hat{\eta}) \times(\hat{\mathrm{V}}, 0, \hat{\xi})$ (cf. $\S 3.1)$.

We say that a real commuting pair $(\eta, \xi)$ with an irrational rotation number has complex a priori bounds, if all its renormalizations extend to holomorphic commuting pairs with bounded moduli:

$$
\bmod (\Delta \backslash \Omega)>\mu>0 \text {. }
$$


For $\mu \in(0,1)$ let $\mathfrak{H}(\mu)$ denote the space of holomorphic commuting pairs $\mathscr{H}$ : $\Omega_{\mathscr{H}} \rightarrow \Delta_{\mathscr{H}}$, with $\bmod \left(\Delta_{\mathscr{H}} \backslash \Omega_{\mathscr{H}}\right)>\mu, \min \left(\left|\mathrm{I}_{\eta}\right|,\left|\mathrm{I}_{\xi}\right|\right)>\mu$ and $\operatorname{diam}\left(\Delta_{\mathscr{H}}\right)<1 / \mu$.

Lemma 5.3 (Lemma 2.15 [Ya2]). - For each $\mu \in(0,1)$ the space $\mathfrak{H}(\mu)$ is sequentially pre-compact, with every limit point contained in $\mathfrak{H}(\mu / 2)$.

The existense of complex a priori bounds is a key analytic issue of renormalization theory. In the case of critical circle maps it is settled by the following theorem:

Theorem 5.4. - There exist universal constants $\mu>0$ and $\mathrm{K}>1$ such that the following holds. Let $\zeta \in \mathfrak{C}$ be a critical commuting pair with an irrational rotation number. Then there exists $\mathrm{N}=\mathrm{N}(\zeta)$ such that for all $n \geq \mathrm{N}$ the commuting pair $\mathscr{R}^{n} \zeta$ extends to a holomorphic commuting pair $\mathscr{H}_{n}: \Omega_{n} \rightarrow \Delta_{n}$ in $\mathfrak{H}(\mu)$. The range $\Delta_{n}$ is a Euclidean disk, and the regions $\Omega_{n} \cap( \pm \mathbf{H})$ are $\mathrm{K}$-quasidisks.

Remark 5.1. - We first proved this theorem in [Yal] for commuting pairs $\zeta$ in an Epstein class $\mathscr{E}_{s}$, in which case $\mathrm{N}=\mathrm{N}(s)$. Our proof was later adapted by de Faria and de Melo [dFdM2] to the case of a non-Epstein critical commuting pair. In the general case, in a Carathéodory compact family of critical commuting pairs, the number $\mathrm{N}$ can be chosen uniformly.

Let $\zeta$ be at least $n$ times renormalizable critical commuting pair. For the lack of a better term, let us say that the pair of numbers $\tau_{n}(\zeta)=\left(r_{n-1}, r_{n-2}\right)$ forms the history of the pair $\mathscr{R}^{n} \zeta$. Based on the above theorem and a detailed analysis of the shapes of the domains $\Omega_{n}$ we proved the following in [Ya1]:

Theorem 5.5 ([Ya1]). - There exists a universal constant $\mathrm{K}_{1}>1$ such that the following holds. Let $\zeta_{1}=\left(\eta_{1}, \xi_{1}\right)$ and $\zeta_{2}=\left(\eta_{2}, \xi_{2}\right)$ be two critical commuting pairs with irrational rotation numbers. Let $n>\max \left(\mathrm{N}\left(\zeta_{1}\right), \mathrm{N}\left(\zeta_{2}\right)\right)+1$ as above. Assume that the $n$-th renormalizations of $\zeta_{1}, \zeta_{2}$ have the same rotation number and the same history. Then their holomorphic commuting pair extensions $\mathscr{H}_{n}^{1}, \mathscr{H}_{n}^{2}$ are $\mathrm{K}_{1}$-quasiconformally conjugate. The conjugating map is conformal on the filled Fulia set.

For commuting pairs of the type bounded by $\mathrm{B}$ this theorem was first proved by de Faria $[\mathrm{dF} 1, \mathrm{dF} 2]$, with " $\mathrm{K}_{1}$ " depending on the value of $\mathrm{B}$.

\subsection{Limiting renormalization towers}

Sullivan's original proof of the existence and uniqueness of the Feigenbaum fixed point used the Teichmüller theory for Riemann surface laminations associated to quadratic-like maps, which he himself developed. McMullen [McM2] 
has replaced this approach with an elegant argument based on a rigidity result for dynamical objects he called renormalization towers. The renormalization towers of critical commuting pairs were studied in [dFdM2] for pairs of bounded type, and later in [Ya2] without the restriction on the type. Their definition is as follows. Let $\left\{\mathscr{H}_{k}\right\}_{1}^{\infty}$ be a sequence of generalized holomorphic commuting pairs. Set $\zeta_{k}=\zeta_{\mathscr{H}_{k}}$. Suppose $m(k)>n(k)$ are two sequences of natural numbers such that $n(k) \rightarrow \infty, m(k)-n(k) \rightarrow \infty$ and $\zeta_{k}$ is $m(k)$-times renormalizable. Moreover, the pre-renormalizations $p \mathscr{R}^{n(k)+i} \zeta_{k}$ have holomorphic pair extensions $\mathscr{H}_{k}^{i}$ such that $\mathscr{H}_{k}^{i} \rightarrow \mathscr{H}_{i} \in \mathfrak{H}$, for $i \in \mathbf{Z}$. Then the bi-infinite sequence $\mathscr{T}=\left(\mathscr{H}_{i}\right)_{-\infty}^{\infty}$ is referred to as a bi-infinite limiting renormalization tower. The rotation number $\rho(\mathscr{T})$ is naturally defined as the bi-infinite sequence of heights $\left(\chi\left(\zeta_{\mathscr{C}_{i}}\right)\right)_{-\infty}^{\infty}$. The precise formulation of Theorem 4.2 is:

Tower Rigidity Theorem ([Ya2]). - For any bi-infinite sequence $\bar{\rho}=\left(\chi_{n}\right)_{-\infty}^{\infty}, \quad \chi_{n} \in$ $\mathbf{N} \cup\{\infty\}$ there exists a bi-infinite limiting renormalization tower $\mathscr{T}$ with $\rho(\mathscr{T})=\bar{\rho}$. Moreover, for any two limiting renormalization towers $\mathscr{T}_{1}=\left(\mathscr{H}_{i}^{1}\right)_{-\infty}^{\infty}, \mathscr{T}_{2}=\left(\mathscr{H}_{i}^{2}\right)_{-\infty}^{\infty}$ with $\rho\left(\mathscr{T}_{1}\right)=$ $\rho\left(\mathscr{T}_{2}\right)$ we have

$$
\zeta_{\mathscr{H}_{i}^{1}} \equiv \zeta_{\mathscr{H}_{i}^{2}} \text { for all } i
$$

\section{Parabolic maps and their perturbations}

General facts. - We begin with a brief review of the theory of parabolic bifurcations, as applied in particular to an interval map in the Epstein class. For a more comprehensive exposition the reader is referred to [Do], supporting technical details may be found in [Sh]. Fix a map $\eta_{0} \in \mathscr{E}$ having a parabolic fixed point $p$ with unit multiplier.

Theorem 6.1 (Fatou Coordinates). — There exist topological discs $\mathrm{U}^{\mathrm{A}}$ and $\mathrm{U}^{\mathrm{R}}$, called attracting and repelling petals, whose union is a punctured neighborhood of the parabolic periodic point $p$ such that

$$
\begin{aligned}
& \eta_{0}\left(\overline{\mathrm{U}}^{\mathrm{A}}\right) \subset \mathrm{U}^{\mathrm{A}} \bigcup\{p\}, \text { and } \bigcap_{k=0}^{\infty} \eta_{0}^{k}\left(\overline{\mathrm{U}}^{\mathrm{A}}\right)=\{p\}, \\
& \eta_{0}\left(\overline{\mathrm{U}}^{\mathrm{R}}\right) \subset \mathrm{U}^{\mathrm{R}} \bigcup\{p\} \text {, and } \bigcap_{k=0}^{\infty} \eta_{0}^{-k}\left(\overline{\mathrm{U}}^{\mathrm{R}}\right)=\{p\},
\end{aligned}
$$

where $\eta_{0}^{-1}$ is the univalent branch fixing $\zeta$.

Moreover, there exist injective analytic maps

$$
\Phi^{\mathrm{A}}: \mathrm{U}^{\mathrm{A}} \rightarrow \mathbf{C} \text { and } \Phi^{\mathrm{R}}: \mathrm{U}^{\mathrm{R}} \rightarrow \mathbf{C},
$$


unique up to post-composition by translations, such that

$$
\Phi^{\mathrm{A}}\left(\eta_{0}(z)\right)=\Phi^{\mathrm{A}}(z)+1 \text { and } \Phi^{\mathrm{R}}\left(\eta_{0}(z)\right)=\Phi^{\mathrm{R}}(z)-1 .
$$

The Riemann surfaces $\mathrm{C}^{\mathrm{A}}=\mathrm{U}^{\mathrm{A}} / \eta_{0}$ and $\mathrm{C}^{\mathrm{R}}=\mathrm{U}^{\mathrm{R}} / \eta_{0}$ are conformally equivalent to the cylinder $\mathbf{C} / \mathbf{Z}$.

Proof. - To fix our ideas, let us assume that $p>0$. We will show that $p$ is a simple parabolic point of $\eta_{0}$, that is,

$$
\eta_{0}(z-p)=(z-p)+a(z-p)^{2}+o\left((z-p)^{2}\right)
$$

with $a \neq 0$. Indeed, for a small $c<0$ consider the map $\eta_{c}=\eta_{0}+c$. As $\eta_{c} \in \mathscr{E}$, there exists a well-defined inverse branch $\phi_{c}$ of this map in $\mathbf{G} \backslash \mathbf{R}$ which preserves both the upper and the lower half-planes. Since $\eta_{c}$ has no real fixed points, the DenjoyWolff Theorem implies that there exists a pair of complex conjugate fixed points $p_{c}^{ \pm} \in \pm \mathbf{H}$ such that every orbit originating in $\mathbf{H}$ converges to $p_{c}^{+}$and similarly for the other fixed point. Thus, $p$ splits into a pair of repelling fixed points, and hence its algebraic multiplicity is two. The rest of the construction is classical.

We denote $\pi_{\mathrm{A}}: \mathrm{U}^{\mathrm{A}} \rightarrow \mathrm{C}^{\mathrm{A}}$ and $\pi_{\mathrm{R}}: \mathrm{U}^{\mathrm{R}} \rightarrow \mathrm{C}^{\mathrm{R}}$ the natural projections. The quotients $\mathrm{C}^{\mathrm{A}}$ and $\mathrm{C}^{\mathrm{R}}$ are customarily referred to as Écalle-Voronin cylinders; we will find it useful to regard these as Riemann spheres with distinguished points,+filling in the punctures. The real axis projects to the natural equators $\mathrm{E}^{\mathrm{A}} \subset \mathrm{C}^{\mathrm{A}}$ and $\mathrm{E}^{\mathrm{R}} \subset \mathrm{C}^{\mathrm{R}}$. Any conformal transit homeomorphism $\tau: \mathrm{C}^{\mathrm{A}} \rightarrow \mathrm{C}^{\mathrm{R}}$ fixing the ends,+is a translation in suitable coordinates. Lifiting it produces a map $\bar{\tau}: \mathrm{U}^{\mathrm{A}} \rightarrow \mathbf{C}$ satisfying

$$
\tau \circ \pi_{\mathrm{A}}=\pi_{\mathrm{R}} \circ \bar{\tau}
$$

We will sometimes write $\tau \equiv \tau_{\theta}$, and $\bar{\tau}=\bar{\tau}_{\theta}$, where

$$
\Phi^{\mathrm{R}} \circ \bar{\tau} \circ\left(\Phi^{\mathrm{A}}\right)^{-1}(z) \equiv z+\theta \bmod \mathbf{Z} .
$$

Suppose for an Epstein map $\eta$ in a sufficiently small neighborhood of $\eta_{0}$ the parabolic point splits into a complex conjugate pair of repelling fixed points $p_{\eta} \in \mathbf{H}$ and $\bar{p}_{\eta}$ with multipliers $\lambda_{\eta}^{ \pm}=e^{2 \pi i \pm \alpha(\eta)}$. In this situation one may still speak of attracting and repelling petals:

Lemma 6.2 (Douady Coordinates). - There exists a neighborhood $\mathrm{U}\left(\eta_{0}\right) \subset \mathscr{E}$ of the map $\eta_{0}$ such that for any $\eta \in \mathrm{U}\left(\eta_{0}\right)$ with $|\arg \alpha(\eta)|<\pi / 4$, there exist topological discs $\mathrm{U}_{\eta}^{\mathrm{A}}$ and $\mathrm{U}_{\eta}^{\mathrm{R}}$ whose union is a neighborhood of $p$, and injective analytic maps

$$
\Phi_{\eta}^{\mathrm{A}}: \mathrm{U}^{\mathrm{A}} \rightarrow \mathbf{G} \text { and } \Phi_{\eta}^{\mathrm{R}}: \mathrm{U}_{f}^{\mathrm{R}} \rightarrow \mathbf{C}
$$


unique up to post-composition by translations, such that

$$
\Phi_{\eta}^{\mathrm{A}}(\eta(z))=\Phi_{\eta}^{\mathrm{A}}(z)+1 \text { and } \Phi_{\eta}^{\mathrm{R}}(\eta(z))=\Phi_{\eta}^{\mathrm{R}}(z)+1 .
$$

The quotients $\mathrm{C}_{\eta}^{\mathrm{A}}=\mathrm{U}_{\eta}^{\mathrm{A}} / \eta$ and $\mathrm{C}_{\eta}^{\mathrm{R}}=\mathrm{U}_{\eta}^{\mathrm{R}} / \eta$ are Riemann surfaces conformally equivalent to $\mathbf{C} / \mathbf{Z}$.

Let us note:

Proposition 6.3. - There exists an open neighborhood $\mathscr{W}\left(\eta_{0}\right) \subset \mathscr{E}_{s}$ of $\eta_{0}$ in the Carathéodory topology, such that for every $\eta \in \mathscr{W}\left(\eta_{0}\right)$ as above, the condition on the eigenvalues of the repelling fixed points is automatically satisfied.

Proof. - Recall that if $z_{0}$ is an isolated fixed point of an analytic map $f$, then the holomorphic index of $z_{0}$ is the residue

$$
i\left(f, z_{0}\right)=\frac{1}{2 \pi i} \oint \frac{d z}{z-f(z)}
$$

where the integrating is done over a small contour enclosing $z_{0}$ (see [Mil]). In the case when $\lambda=f^{\prime}(z) \neq 1$, an elementary computation shows that $i\left(f, z_{0}\right)=$ $1 /(1-\lambda)$. In our case, by continuity,

$$
\frac{1}{1-\lambda_{\eta}^{+}}+\frac{1}{1-\lambda_{\eta}^{-}} \underset{\eta \rightarrow \eta_{0}}{\longrightarrow} i\left(\eta_{0}, p\right) \text {. }
$$

Since the right-hand side is finite, and $\lambda_{\eta}^{+}=\overline{\lambda_{\eta}^{-}}$, and $1-\lambda_{\eta}^{ \pm} \underset{\eta \rightarrow \eta_{0}}{\longrightarrow} 0$, it follows that

$$
\left|\arg \left(1-\lambda_{\eta}^{ \pm}\right)\right| \underset{\eta \rightarrow \eta_{0}}{\longrightarrow} \frac{\pi}{2}
$$

An arbitrary choice of real basepoints $a \in \mathrm{U}^{\mathrm{A}}$ and $r \in \mathrm{U}^{\mathrm{R}}$ enables us to specify the Fatou and Douady coordinates uniquely, by requiring that $\Phi^{\mathrm{A}}(a)=$ $\Phi_{\eta}^{\mathrm{A}}(a)=0$, and $\Phi^{\mathrm{R}}(r)=\Phi_{\eta}^{\mathrm{R}}(r)=0$. The following fundamental theorem first appeared in [DH1]:

Theorem 6.4. - With these normalizations the maps $\Phi_{\eta}^{\mathrm{A}}, \Phi_{\eta}^{\mathrm{R}}$ depend continuously on $\eta$ with respect to the compact-open topology, and

$$
\Phi_{\eta}^{\mathrm{A}} \rightarrow \Phi^{\mathrm{A}} \text { and } \Phi_{\eta}^{\mathrm{R}} \rightarrow \Phi^{\mathrm{R}}
$$

uniformly on compact subsets of $\mathrm{U}^{\mathrm{A}}$ and $\mathrm{U}^{\mathrm{R}}$ respectively.

Moreover, select the smallest $n(\eta) \in \mathbf{N}$ for which $\eta^{n(\eta)}(a) \geq r$. Then

$$
\eta^{n(\eta)}(z)=\left(\Phi_{\eta}^{\mathrm{R}}\right)^{-1} \circ \mathrm{T}_{\theta(\eta)+\mathrm{K}} \circ \Phi_{\eta}^{\mathrm{A}}
$$


wherever both sides are defined. In this formula $\mathrm{T}_{a}(z)$ denotes the translation $z \mapsto z+a$, $\theta(\eta) \in[0,1)$ is given by

$$
\theta(\eta)=1 / \alpha(\eta)+\underset{\alpha(\eta) \rightarrow \infty}{o(1)} \bmod 1
$$

and the real constant $\mathrm{K}$ is determined by the choice of the basepoints $a, r$. Thus for a sequence $\left\{\eta_{k}\right\} \subset \mathrm{U}(\eta)$ converging to $\eta$, the iterates $\eta_{k}^{n\left(\eta_{k}\right)}$ converge locally uniformly if and only if there is a convergence $\theta(\eta) \rightarrow \theta$, and the limit in this case is a certain lift of the transit homeomorphism $\tau_{\theta}$ for the parabolic map $\eta_{0}$.

As a final remark in this section, let us note that, for example, Shishikura states Lemma 6.2 and Theorem 6.4 [Sh] for an open set of maps in a Banach manifold, and the proofs he gives actually imply that Douady's coordinates vary locally analytically with the map $\eta$. We will prove a similar statement further in this paper.

\section{Renormalization of maps of the cylinder}

\subsection{A space of maps of the cylinder and a renormalization transformation}

In this section we will introduce a new renormalization procedure defined on a space of analytic maps of the cylinder. As it will turn out this renormalization is naturally related to the renormalization of critical circle maps, and has some important advantages over the latter. Firstly, let us denote $\pi$ the natural projection $\mathbf{G} \rightarrow \mathbf{G} / \mathbf{Z}$. For an equatorial annulus $U \subset \mathbf{G} / \mathbf{Z}$ let $\mathfrak{A}_{\mathrm{U}}$ be the space of bounded analytic maps $\phi: \mathrm{U} \rightarrow \mathbf{C} / \mathbf{Z}$, such that $\phi(\mathbf{T})$ is homotopic to $\mathbf{T}$, equipped with the uniform metric. We shall turn $\mathfrak{A}_{\mathrm{U}}$ into a real-symmetric complex Banach manifold as follows. Denote $\tilde{U}$ the lift $\pi^{-1}(\mathrm{U}) \subset \mathbf{C}$. The space of functions $\tilde{\phi}: \tilde{U} \rightarrow \mathbf{C}$ which are analytic, continuous up to the boundary, and 1-periodic, $\tilde{\phi}(z+1)=\tilde{\phi}(z)$, becomes a Banach space when endowed with the sup norm. Denote that space $\tilde{\mathfrak{A}}_{\mathrm{U}}$. For a function $\phi: U \rightarrow \mathbf{C} / \mathbf{Z}$ denote $\check{\phi}$ an arbitrarily chosen lift $\pi\left(\check{\phi}\left(\pi^{-1}(z)\right)\right)=\phi$. Observe that $\phi \in \mathfrak{A}_{\mathrm{U}}$ if and only if $\tilde{\phi}=\check{\phi}-\operatorname{Id} \in \tilde{\mathfrak{A}}_{\mathrm{U}}$. We use the local homeomorphism between $\tilde{\mathfrak{A}}_{\mathrm{U}}$ and $\mathfrak{A}_{\mathrm{U}}$ given by

$$
\tilde{\phi} \mapsto \pi \circ(\tilde{\phi}+\mathrm{Id}) \circ \pi^{-1}
$$

to define the atlas on $\mathfrak{A}_{\mathrm{U}}$. The coordinate change transformations are given by $\tilde{\phi}(z) \mapsto \tilde{\phi}(z+n)+m$ for $n, m \in \mathbf{Z}$, therefore with this atlas $\mathfrak{A}_{\mathrm{U}}$ is a real-symmetric complex Banach manifold.

Now let $f: \mathbf{U} \rightarrow \mathbf{G} / \mathbf{Z}$ be an analytic critical circle map. By definition, there is a neighborhood of the equator in which 0 is the only critical point of $f$. Let $\tilde{f}: \tilde{U} \rightarrow \mathbf{C}$ be a lift of $f$. The Argument Principle implies that for $\epsilon>0$ small 
enough, if $\tilde{g} \in \tilde{\mathfrak{A}}_{\mathrm{U}}$ is real-analytic, with $\tilde{g}^{\prime}(0)=-1, \tilde{g}^{\prime \prime}(0)=0$, and $\|\tilde{f}-\tilde{g}\|<\epsilon$, then $g=\pi \circ(\tilde{g}+\mathrm{Id}) \circ \pi^{-1}$ is a critical circle map as well. Let $\epsilon(f)$ be the supremum of such $\epsilon$, and set

$$
\begin{gathered}
\mathfrak{C}_{\mathrm{U}}=\cup_{f}\left\{\pi \circ(\tilde{g}+\mathrm{Id}) \circ \pi^{-1} \mid \tilde{g} \in \tilde{\mathfrak{A}}_{\mathrm{U}}, \tilde{g}^{\prime}(0)=-1, \tilde{g}^{\prime \prime}(0)=0,\right. \\
\text { and }\|\tilde{g}-\tilde{f}\|<\epsilon(f)\} .
\end{gathered}
$$

Proposition 7.1. - The space $\mathfrak{C}_{\mathrm{U}}$ is a codimension 2 submanifold of $\mathfrak{A}_{\mathrm{U}}$.

Proof. - Set

$$
\mathfrak{B}_{\mathrm{U}}=\left\{\tilde{\phi} \in \tilde{\mathfrak{A}}_{\mathrm{U}} \text { with } \tilde{\phi}^{\prime}(0)=0, \tilde{\phi}^{\prime \prime}(0)=0\right\} .
$$

This is a Banach subspace of $\tilde{\mathfrak{A}}_{\mathrm{U}}$, which has codimension 2 by the Implicit Function Theorem. Select an element $\psi \in \tilde{\mathfrak{A}}_{\mathrm{U}}$ with $\psi^{\prime}(0)=-1, \psi^{\prime \prime}(0)=0$. In the local chart on $\mathfrak{A}_{\mathrm{U}}$ defined by

$$
\tilde{\phi} \mapsto \pi \circ(\tilde{\phi}+\mathrm{Id}) \circ \pi^{-1}
$$

the image of $\mathfrak{C}_{U}$ is the affine subspace $\psi+\mathfrak{B}_{U}$.

We shall say that $f$ is a critical cylinder map if $f \in \mathfrak{C}_{\mathrm{U}}$ for some $\mathrm{U} \subset \mathbf{C} / \mathbf{Z}$. Let us denote by $\mathfrak{C}_{\mathrm{U}}^{\mathbf{R}}$ the real slice of $\mathfrak{C}_{\mathrm{U}}$ :

$$
\begin{aligned}
\mathfrak{C}_{\mathrm{U}}^{\mathbf{R}} & =\left\{f \in \mathfrak{C}_{\mathrm{U}} \text { with } \bar{f}(z)=f(\bar{z})\right\} \\
& =\left\{f \in \mathfrak{A}_{\mathrm{U}}, \text { such that }\left.f\right|_{\mathbf{T}} \text { is a critical circle map }\right\} .
\end{aligned}
$$

Definition 7.1. - Given a critical cylinder map $f \in \mathfrak{C}_{\mathrm{U}}$ let us say that it is cylinder renormalizable, or simply renormalizable, if there exists $k>1$ and an equatorial annulus $\mathrm{V} \subset \mathbf{C} / \mathbf{Z}$ such that following holds:

- there exist repelling periodic points $p_{1}, p_{2}$ of $f$ in $\mathrm{U}$ with periods $k$ and a simple arc $l$ connecting them such that $f^{k}(l)$ is a simple arc, and $f^{k}(l) \cap l=\left\{p_{1}, p_{2}\right\}$;

- the iterate $f^{k}$ is defined and univalent in the domain $\mathrm{C}_{f}$ bounded by $l$ and $f^{k}(l)$, the corresponding inverse branch $\left.f^{-k}\right|_{f^{k}\left(\mathrm{C}_{f}\right)}$ univalently extends to $\mathrm{C}_{f}$; and the quotient of $\overline{\mathrm{C}_{f} \cup f^{k}\left(\mathrm{C}_{f}\right)} \backslash\left\{p_{1}, p_{2}\right\}$ by the action of $f^{k}$ is a Riemann surface conformally isomorphic to the cylinder $\mathbf{C} / \mathbf{Z}$ (we will call a domain $\mathbf{C}_{f}$ with these properties a fundamental crescent of $\left.f^{k}\right)$;

- for a point $z \in \overline{\mathrm{C}}_{f}$ with $\left\{f^{j}(z)\right\}_{j \in \mathbf{N}} \cap \overline{\mathrm{C}}_{f} \neq \varnothing$, set $\mathrm{R}_{\mathrm{C}_{f}}(z)=f^{n(z)}(z)$ where $n(z) \in \mathbf{N}$ is the smallest value for which $f^{n(z)}(z) \in \overline{\mathbf{C}}_{f}$. We further require that there exists a point $c$ in the domain of $\mathbf{R}_{\mathrm{C}_{f}}$ such that $f^{m}(c)=0$ for some $m<n(c)$; and if we denote $\hat{f}$ the projection of $\mathbf{R}_{\mathrm{C}_{f}}$ to $\mathbf{G} / \mathbf{Z}$ with $c \mapsto 0$, then $\hat{f} \in \mathfrak{C}_{\mathrm{V}}$.

We will say that the new critical circle map $\hat{f}$ is a cylinder renormalization of $f$ with period $k$. 
The relation of the cylinder renormalization procedure to critical circle maps is easy to see:

Proposition 7.2. - Suppose $f \in \mathfrak{C}_{\mathrm{U}}$ is a critical circle map with rotation number $\rho(f) \in \mathbf{R} \backslash \mathbf{Q}$. Assume that it is cylinder renormalizable with period $q_{n}$. Then the corresponding renormalization $\hat{f}$ is also a critical circle map with rotation number $\mathrm{G}^{n}(\rho(f))$. Also, $\mathscr{R} \hat{f}$ is analytically conjugate to $\mathscr{R}^{n+1} f$.

Proof. - As follows from the definition of a fundamental crescent, the intersection of the domain $\mathbf{C}_{f}$ with $\mathbf{T}$ is an arc of the form $\left[a, f^{q_{n}}(a)\right]$. The claim follows in an obvious fashion from this observation.

The first useful property of cylinder renormalization is the following:

Proposition 7.3. - Let $f \in \mathfrak{C}_{\mathrm{U}}$ be renormalizable, with a cylinder renormalization $\hat{f} \in \mathfrak{C}_{\mathrm{V}}$ corresponding to the fundamental crescent $\mathrm{C}_{f}$, and let $\mathrm{W}$ be any equatorial annulus compactly contained in $\mathrm{V}$. Then there is an open neighborhood $\mathrm{G} \subset \mathfrak{C}_{\mathrm{U}}$ of $f$ such that every $g \in \mathrm{G}$ is renormalizable, with a fundamental crescent $\mathrm{C}_{g} \subset \mathrm{U}$ which depends continuously on $g$ in the Hausdorff sense. Moreover, there exists a holomorphic motion $\chi_{g}: \partial \mathrm{C}_{f} \mapsto \partial \mathrm{G}_{g}$ over $\mathrm{G}$, such that $\chi_{g}(f(z))=g\left(\chi_{g}(z)\right)$. And finally, the renormalization $\hat{g}$ is contained in $\mathfrak{C}_{W}$.

Proof. - Firstly, there is a neighborhood of $f$ in which there exist continuous branches of $g^{k}$-periodic points $p_{1}^{g}, p_{2}^{g}$ with $p_{i}^{f}=p_{i}$, such that both points are repelling. Shrinking the neighborhood, if necessary, we may select an analytic family of linearizing coordinates $w_{i}^{g}: \mathrm{D}_{i}^{g} \rightarrow \mathbf{D}$ which conjugate the iterate $g^{k}$ to the linear map $z \mapsto \lambda_{i}^{g} z$, with $\lambda_{i}^{g}=\left(g^{k}\right)^{\prime}\left(p_{i}^{g}\right)$ (here $\mathrm{D}_{i}^{g}$ is a topological disk around $p_{i}^{g}$, and $\left.w_{i}^{g}\left(p_{i}^{g}\right)=0\right)$. We may further assume that $w_{i}^{g}\left(\mathrm{D}_{i}^{g}\right)=\mathbf{D}$. Suppose $\mathrm{G}_{f}$ is bounded by the curves $l_{f}, f^{k}\left(l_{f}\right)$ terminating at the points $p_{i}^{f}$, and let us denote $z_{i}$ the points in $\partial \mathrm{D}_{i}^{f} \cap f^{k}\left(l_{f}\right)$ which are obtained last, when moving along $f^{k}\left(l_{f}\right)$ starting from the equator, and $\zeta_{i}$ their preimages by the branches of $f^{-k}$ defined in $\mathrm{D}_{i}^{f}$ and fixing $p_{i}^{f}$. Note that removing a smooth simple curve $\gamma_{i}^{f} \subset \mathrm{D}_{i}^{f}$ connecting $p_{i}^{f}$ to the boundary of $\mathrm{D}_{i}^{f}$ from $\mathrm{D}_{i}^{f}$ we may select a branch of

$$
\mathrm{W}_{i}^{f}=\frac{1}{\log \lambda_{i}^{g}} \log w_{i}^{g}
$$

conjugating $f^{k}$ to $z \mapsto z+1$ in the complementary domain. Let us embed $\gamma_{i}^{f}$ into an analytic family of curves $\gamma_{i}^{g}$ with the same properties, and define $\mathrm{W}_{i}^{g}$ in a similar fashion, selecting the same branch of log for all maps in a neighborhood of $f$.

Now we are prepared to define $\mathrm{C}_{g}$. Let $l$ be the segment of $l_{f}$ terminating at the points $\zeta_{1}, \zeta_{2}$, and $l_{i}$ the complementary segment containing $p_{i}^{f}$. Now let 
$\mathrm{L}_{i}$ be the curve $l_{i}$ in $\mathrm{W}_{i}^{f}$-coordinates, and $\mathrm{L}_{i}^{g}$ the parallel translate of this curve terminating at $\mathrm{W}_{i}^{g}\left(\zeta_{i}\right)$. We set $l_{g}$ to be the union of three curves: $l$, and $l_{i}^{g}=$ $\left(\mathrm{W}_{i}^{g}\right)^{-1}\left(\mathrm{~L}_{i}^{g}\right)$, and define $l_{g}^{\prime}=g^{k}\left(l_{g}\right)$. By construction, shrinking the neighborhood around $f$ again, if necessary, we may ensure that the curves $l_{g}$, $l_{g}^{\prime}$ are disjoint away from the ends. Denote $\mathrm{C}_{g}$ the domain bounded by $l_{g}, l_{g}^{\prime}$, chosen so that $g \mapsto \mathrm{C}_{g}$ is Hausdorff continuous. By the Grötzsch inequality, $\mathrm{C}_{g}{ }^{3}$ is a fundamental crescent for $g$. The existense of the desired holomorphic motion is obvious from the construction.

It remains to show that $\mathrm{G}$ may be chosen so that $\hat{g} \in \mathfrak{C}_{\mathrm{W}}$. It clearly follows from Definition 7.1 that there is a domain $\mathrm{D} \subset \mathrm{C}_{f} \cup f^{-k}\left(\mathrm{C}_{f}\right)$ and an iterate $n>k$ such that $\mathrm{W} \Subset \pi_{f}(\mathrm{D})$ (where $\pi_{f}: \mathrm{C}_{f} \rightarrow \mathbf{G} / \mathbf{Z}$ denotes the appropriate projection) and $\hat{f} \in \mathfrak{C}_{\mathrm{W}}$ is the projection of the iterate $\left.f^{n}\right|_{\mathrm{D}}$. Now the iterate $\left.g^{n}\right|_{\mathrm{D}}$ projects to an analytic map $\hat{g}$ which is in $\mathfrak{C}_{\mathrm{W}}$ for all $g$ sufficiently close to $f$.

The next proposition shows that $g \mapsto \hat{g}$ is well-defined:

Proposition 7.4. - In the notations of of Proposition 7.3, let $\mathrm{C}_{g}^{\prime}$ be a different family of fundamental crescents, which also depends continuously on $g \in \mathrm{G}$ in the Hausdorff sense. Then there exists an open neighborhood $\mathrm{G}^{\prime} \subset \mathrm{G}$ of $f$, such that the cylinder renormalization of $g \in \mathrm{G}^{\prime}$ corresponding to $\mathrm{C}_{g}^{\prime}$ is also $\hat{g}$.

Proof. - The argument is standard in the study of Douady coordinates; we outline it, and leave the details to the reader. As we have seen above, in a slit neighborhood of $p_{i}^{g}$, the iterate $g^{k}$ is conjugate to $z \mapsto z+1$, and both $\mathrm{C}_{g}$ and $\mathrm{C}_{g}^{\prime}$ become fundamental half-infinite strips for the orbits of the unit translation. Let us define a projection $\pi: \mathrm{C}_{g} \rightarrow \mathrm{C}_{g}^{\prime}$ as follows: in a small neighborhood of the repelling point $p_{i}^{g}$ the point $z \in \mathrm{C}_{g}$ will project to a point $z^{\prime} \in \mathrm{C}_{g}^{\prime}$, which is its integer translate in the log-linearizing coordinate. Outside the linearizing neighborhoods of the repelling points, every $z \in \mathrm{C}_{g}$ belongs to a union $\mathrm{C}_{g} \cup f^{k}\left(\mathrm{C}_{g}\right) \cup\left(\left.f^{k}\right|_{\mathrm{C}_{g}}\right)^{-1}\left(\mathrm{C}_{g}\right)$ (provided $\mathrm{G}^{\prime}$ is chosen small enough). We then set $\pi(z)$ to be one of the points $z, f^{k}(z),\left(\left.f^{k}\right|_{\mathrm{C}_{g}}\right)^{-1}(z)$ which belongs to $\mathrm{C}_{g}^{\prime}$. In the quotient cylinders, $\pi$ becomes a conformal map $\mathbf{G} / \mathbf{Z} \rightarrow \mathbf{G} / \mathbf{Z}$ which conjugates the corresponding cylinder renormalizations of $g$. However, it also fixes $0 \in \mathbf{C} / \mathbf{Z}$ and hence is the identity.

We invite the reader to verify, along the same lines, that if $t \mapsto \mathrm{C}_{f}^{t}$ is a homotopy of fundamental crescents for $f$, then the corresponding cylinder renormalizations all coincide. However, a critical cylinder map may be renormalizable in several different ways. Further in this section we will make a canonical choice of cylinder renormalization for a particular class of critical cylinder maps.

A key property of the cylinder renormalization is the following: 
Proposition 7.5. - In the notations of Proposition 7.3, the dependence $g \mapsto \hat{g}$ is an analytic map $\mathrm{G} \rightarrow \mathfrak{C}_{\mathrm{W}}$.

Proof. - By the Theorem of Bers and Royden [BR], the holomorphic motion $\chi_{g}$ extends to a holomorphic motion $\chi_{g}: \mathrm{C}_{f} \cup f^{k}\left(\mathrm{C}_{f}\right) \rightarrow \mathrm{C}_{g} \cup g^{k}\left(\mathrm{C}_{g}\right)$ over a smaller open neighborhood $\mathrm{G}^{\prime}$ with the same equivariance property. As shown in [MSS], the holomorphic motion induces an analytic family of quasiconformal maps

$$
\Psi_{g}: \mathbf{C} / \mathbf{Z}=\left(\mathrm{C}_{f} \cup f^{k}\left(\mathrm{C}_{f}\right)\right) / f^{k} \rightarrow \mathbf{C} / \mathbf{Z}=\left(\mathrm{C}_{g} \cup g^{k}\left(\mathrm{C}_{g}\right)\right) / g^{k} .
$$

Applying the theorem of Ahlfors and Bers, we see that the projection $\pi_{g}: \overline{\mathrm{C}_{g}} \cup$ $g^{k}\left(\mathbf{C}_{g}\right) \rightarrow \mathbf{C} / \mathbf{Z}$ depends analytically on $g$. Let $\mathrm{D} \subset \mathrm{C}_{f} \cup f^{-k}\left(\mathrm{C}_{f}\right)$ and $n \in \mathbf{N}$ be such that $\mathrm{W} \Subset \pi_{f}(\mathrm{D})$, and $\hat{f} \in \mathfrak{C}_{\mathrm{W}}$ is the projection of the iterate $\left.f^{n}\right|_{\mathrm{D}}$. The iterate $\left.g^{n}\right|_{\mathrm{D}}$ projects to $\pi_{g} \circ g^{n}=\hat{g} \in \mathfrak{C}_{\mathrm{W}}$ for all $g$ sufficiently close to $f$, and the claim follows.

Let us summarize. The cylinder renormalization acts on critical circle maps themselves, rather than on commuting pairs. The reason for introducing commuting pairs in the first place, was the absense of a canonical affine structure on the smooth circle obtained by glueing the ends of a fundamental domain of the iterate $f^{q_{n+1}}$. However, if $f$ is cylinder renormalizable with period $q_{n}$, the affine structure on the quotient circle comes from the quotient $\mathrm{C}_{f} / f^{q_{n}} \simeq \mathbf{C} / \mathbf{Z}$. The key here is a fundamental fact of complex analysis, known under a slightly different guise as Liouville's Theorem: an analytic manifold structure on $\mathbf{G} / \mathbf{Z}$ is necessarily affine. The second advantage of $\mathscr{R}_{\text {cyl }}$ over $\mathscr{R}$ is closely related to the first one: as we have seen, it extends to analytic maps which are not symmetric with respect to the circle, thereby becoming an analytic operator on a complex Banach manifold. In the following section we will show that Epstein critical circle maps are always cylinder renormalizable.

7.2. Cylinders of commuting pairs in the Epstein class

In this section we will relate the cylinder renormalization defined above with the renormalization of critical commuting pairs in the Epstein class. The crucial observation is the following:

Lemma 7.6. - Let $\zeta=(\eta, \xi)$ be a commuting pair in the Epstein class with $\chi(\zeta) \neq \infty$. Denote $\mathrm{D}_{\eta}$ the domain of the branched triple covering map $\eta: \mathrm{D}_{\eta} \rightarrow \mathbf{C}_{\eta\left(\mathrm{I}_{\eta}\right)}$. Then the following holds:

- there exist two compex conjugate fixed points $p_{\eta}^{+}, p_{\eta}^{-}$of the map $\eta$ with $p_{\eta}^{ \pm} \in \pm \mathbf{H}$, and an $\mathbf{R}$-symmetric simple arc $l$ connecting these points such that $l \subset \mathrm{D}_{\eta}$ and $\eta(l) \cap l=\left\{p_{\eta}^{+}, p_{\eta}^{-}\right\}$ 
- denoting $\mathrm{C}_{\zeta}$ the $\mathbf{R}$-symmetric domain bounded by $l$ and $\eta(l)$ we have $\mathrm{C}_{\zeta} \subset \mathrm{D}_{\eta}$, and the quotient of $\overline{\mathrm{C}_{\zeta} \cup \eta\left(\mathrm{C}_{\zeta}\right)} \backslash\left\{p_{\eta}^{+}, p_{\eta}^{-}\right\}$by the action of $\zeta$ is a Riemann surface homeomorphic to the cylinder $\mathbf{C} / \mathbf{Z}$ (that is, $\mathbf{C}_{\zeta}$ is a fundamental crescent of $\eta$ );

- the dependence $\zeta \mapsto \mathrm{C}_{\zeta}$ is continuous with respect to the Carathéodory topology on the Epstein class and the Hausdorff topology on the image.

Proof. - Let us fix $s>0$ such that $\zeta \in \mathscr{E}_{s}$. In view of Lemma 3.2, there exists $\mathrm{N}=\mathrm{N}(s)$ such that every pair $\zeta \in \mathscr{E}_{s}$ with $\chi(\zeta)>\mathrm{N}$ satisfies the conditions of the existence of Douady coordinates (Lemma 6.2). We may choose as $\mathrm{C}_{\zeta}$ a fundamental domain for $\left.\eta\right|_{\mathrm{U}_{\mathrm{A}}}$, by Theorem 6.4 the choice can be made continuously.

Let us address the case $\chi(\zeta) \leq \mathrm{N}(s)$. Let $\eta^{-1}: \mathbf{H} \rightarrow \mathbf{H}$ denote the inverse branch which preserves the real axis, $\eta^{-1}(\mathbf{H}) \subsetneq \mathbf{H}$. By the Denjoy-Wolf Theorem this branch has a unique fixed point $p_{\eta}^{+} \in \mathbf{H} \cup \eta\left(\mathrm{I}_{\eta}\right)$ which is globally attracting in $\mathbf{H}$. In this case necessarily $\operatorname{Im} p_{\eta}^{+}>0$. Extend $\eta^{-1}$ to $\mathbf{- H}$ by symmetry. This branch has a complex-conjugate fixed point $p_{\eta}^{-}=\overline{p_{\eta}^{+}}$. In view of their uniqueness, these points depend continuously on the map $\eta$. Note that for a converging sequence of commuting pairs with $\chi(\eta) \rightarrow \infty$ these fixed points converge to a parabolic fixed point on the real axis.

Suppose that the commuting pair $\zeta$ does not possess a fundamental crescent $\mathrm{C}_{\zeta}$. As noted in the proof of Proposition 7.3, in a neighborhood of $p_{\eta}^{ \pm}$the map $\eta$ becomes a translation in log-linearizing coordinate. Therefore if $l$ is a curve connecting the fixed points $p_{\eta}^{ \pm}$and the image $\eta(l) \backslash\left\{p_{\eta}^{ \pm}\right\}$is disjoint from $l$, then $l$ can be modified near the ends in such a way that the quotient of the crescent bounded by $l, \eta(l)$ has the conformal type of a bi-infinite cylinder. Thus, for every choice of an $\mathbf{R}$-symmetric curve $l$ connecting the fixed points $p_{\eta}^{ \pm}$the image $\eta(l) \backslash\left\{p_{\eta}^{ \pm}\right\}$intersects with $l$. To fix the ideas, let $\xi(0)>0$. Consider the maps $\eta_{\alpha} \in \mathscr{E}$ given by $\eta_{\alpha}=\eta+\alpha$ for $\alpha>0$. Let $\alpha_{0}$ be the smallest value for which the map $\eta_{\alpha_{0}}$ has a fixed point, which is then necessarily parabolic. For all $\alpha<\alpha_{0}$ sufficiently close to $\alpha_{0}$ the map $\eta_{\alpha}$ has a fundamental crescent by Lemma 6.2. Therefore there exists a value $\alpha_{*}$ between 0 and $\alpha_{0}$ such that $\eta_{*}=\eta_{\alpha_{*}}$ has the following property. For any simple $\mathbf{R}$-symmetric arc $l$ connecting the fixed points of $\eta_{*}$ the intersection $\left(\eta_{*}^{-1}(l) \backslash\left\{p_{\zeta_{*}}^{ \pm}\right\}\right) \cap l$ is non-empty, and there is a curve $\hat{l}$ which touches $\eta_{*}^{-1}(\hat{l})$ without crossing it.

Consider such a curve $\hat{l}$ and denote $\gamma=\hat{l} \cap \eta_{*}^{-1}(\hat{l}) \backslash\left\{p_{\eta_{*}}^{ \pm}\right\}$. Again appealing to the local picture of the dynamics near the fixed points and near the real axis we see that we may choose $\hat{l}$ for which the set $\gamma$ is positive distance away from $p_{\eta_{*}}^{ \pm}$and $\mathbf{R}$. Let us now fix $\hat{l}$ for which $\gamma$ has the smallest Euclidean diameter. Enclose $\gamma$ into a Euclidean disk $\mathrm{E}$ with a diameter equal to diam $\gamma$, and take a point $x \in \gamma \cap \partial \mathrm{E}$. Let $x_{-1}, x_{-2}, \ldots$ denote the consecutive $\eta_{*}$-preimages of $x$ lying 
in $\gamma$. Note that this sequence has to be finite, since otherwise the set $\overline{U x_{-i}} \subset \gamma$ is compact and invariant under $\eta_{*}^{-1}$ and thus contains a fixed point, which contradicts the fact that $p_{\eta_{*}}^{ \pm}$are the only fixed points of $\eta_{*}^{-1}$. Let $x_{-n}$ be the last term in this sequence. Since $\eta_{*}^{-1}\left(x_{-n}\right) \notin \gamma$, we may slightly modify the curve $\hat{l}$ near $x_{-n}$, so that the new curve and its preimage become disjoint in a neighborhood of $x_{-n}$. Proceeding in this fashion, we obtain a curve $\tilde{l}$ which is a small perturbation of $\hat{l}$ such that $\tilde{l} \cap \eta_{*}^{-1}(\tilde{l}) \subset \mathrm{E} \backslash \mathrm{D}_{\epsilon}(x)$ for some $\epsilon>0$. Repeating the procedure a finite number of times we arrive at a curve $\breve{l}$ with

$$
\operatorname{diam}\left(\check{l} \cap \eta_{*}^{-1}(\check{l}) \backslash\left\{p_{\eta_{*}}^{ \pm}\right\}\right)<\operatorname{diam} \gamma
$$

contradictory to the way in which $\breve{l}$ was selected. Tracing back the assumptions, we see that for any $\zeta \in \mathscr{E}$ with $\chi(\zeta) \neq \infty$ the arc $l$ may be chosen with $\eta(l) \cap$ $l=\left\{p_{\eta}^{ \pm}\right\}$. The continuity of the construction is ensured in the same way as in Proposition 7.3.

Given $\zeta=(\eta, \xi) \in \mathscr{E}$ and $\mathrm{C}_{\zeta}$ as above, denote $\mathrm{R}_{\mathrm{C}_{\zeta}}(z)$ the first return map of the crescent $\mathrm{C}_{\zeta}$. Let $c_{\zeta} \in \mathbf{T}$ be the only critical point of $\mathrm{R}_{\mathrm{C}_{\zeta}}$ and denote $\pi_{\mathrm{C}_{\zeta}}: \overline{\mathrm{C}_{\zeta} \cup \eta\left(\mathrm{C}_{\zeta}\right)} \rightarrow \mathbf{C} / \mathbf{Z}$ the projection specified by $c_{\zeta} \mapsto 0$. In an obvious fashion we have:

Proposition 7.7. - The projection of the first return map

$$
f_{\mathrm{C}_{\zeta}}=\pi_{\mathrm{C}_{\zeta}} \circ \mathrm{R}_{\mathrm{C}_{\zeta}} \circ \pi_{\mathrm{C}_{\zeta}}^{-1}
$$

is defined and analytic on an open neighborhood of the circle $\mathbf{T}$, and $\left.f_{\mathrm{C}_{\zeta}}\right|_{\mathbf{T}}$ is an analytic critical circle map.

Let us show that this critical circle map does not, in fact, depend on the particular choice of the fundamental crescent $\mathrm{C}_{\zeta}$ :

Proposition 7.8. - Let $\mathrm{C}_{\zeta}^{\prime} \subset \mathrm{D}_{\zeta}$ be a different domain satisfying the conditions of Lemma 7.6. Then

$$
\left.\left.f_{\mathrm{C}_{\zeta}}\right|_{\mathbf{T}} \equiv f_{\mathrm{C}_{\zeta}^{\prime}}\right|_{\mathbf{T}}
$$

Proof. - To fix our ideas, assume that $\xi(0)>0$. Denote $\mathrm{O} \subset \mathbf{G}$ the smallest full set in the plane containing $\mathrm{C}_{\zeta} \cup \mathrm{C}_{\zeta}^{\prime}$. The set $\mathrm{O}$ is bounded by two curves $l_{1}$, $l_{2}$ meeting at $p_{\zeta}^{ \pm}$, let $l_{1} \cap \mathbf{R}<l_{2} \cap \mathbf{R}$. By construction, the curves $l_{1}$ and $\eta^{-1}\left(l_{1}\right)$ bound a fundamental crescent $\mathrm{C}_{\zeta}^{\prime \prime}$. The Denjoy-Wolff Theorem together with the dynamics of $\left.\eta\right|_{\mathbf{R}}$ implies that for every point $z \in \mathrm{C}_{\zeta}^{\prime \prime}$ there is the smallest $n(z) \geq 0$ such that $\eta^{-n(z)}(z) \in \mathrm{C}_{\zeta}$. Define $h(z) \equiv \eta^{-n(z)}(z)$. The value of $n(z)$ is constant on 
an open neighborhood of $z$ except for $z \in \eta^{n(z)}\left(\partial \mathrm{C}_{\zeta}\right)$ for which the values on the two sides of $\partial \mathrm{C}_{\zeta}$ differ by 1 . Therefore the projection $\psi=\pi_{\mathrm{C}_{\zeta}} \circ h \circ \pi_{\mathrm{C}_{\zeta}^{\prime \prime}}^{-1}$ is an analytic endomorphism of $\mathbf{G} / \mathbf{Z}$. Since $\eta^{-1}$ is univalent, $\psi$ has no critical points, and since $\eta_{\mathbf{R}}$ is monotone, the degree of $\left.\psi\right|_{\mathbf{T}}$ is one. Hence, $\psi$ is a conformal map. Also, $\psi(0)=0$ by construction of the projections $\pi_{\mathrm{C}_{\zeta}}$ and $\pi_{\mathrm{C}_{\zeta}^{\prime \prime}}$. Therefore, $\psi \equiv$ Id. By definition of the first return map, $\psi \circ f_{\mathrm{C}_{\zeta}^{\prime \prime}} \circ \psi^{-1}=f_{\mathrm{C}_{\zeta}}$ on a neighborhood of $\mathbf{T}$, so $f_{\mathrm{C}_{\zeta}^{\prime \prime}}=f_{\mathrm{C}_{\zeta}}$ in that neighborhood. The equality $f_{\mathrm{C}_{\zeta}^{\prime \prime}}=f_{\mathrm{C}_{\zeta}^{\prime}}$ is shown in the same way, and the claim follows.

In view of the last lemma, on a neighborhood of $\mathbf{T}$ we obtain a well-defined analytic critical circle map

$$
f_{\zeta} \equiv f_{\mathrm{C}_{\zeta}},
$$

which we will refer to as the cylinder map of $\zeta$. In Figure 4 we illustrate the action of the map $f_{\zeta}$ near $\mathbf{T}$. Schematically shown is the preimage of the circle $\mathbf{T} \subset \mathbf{G} / \mathbf{Z}$ which consist of the circle itself, together with analytic arcs emanating from 0 at angles $k \pi / 3$ to $\mathbf{T}$. Analytic continuations of these preimages may eventually branch further, as shown.

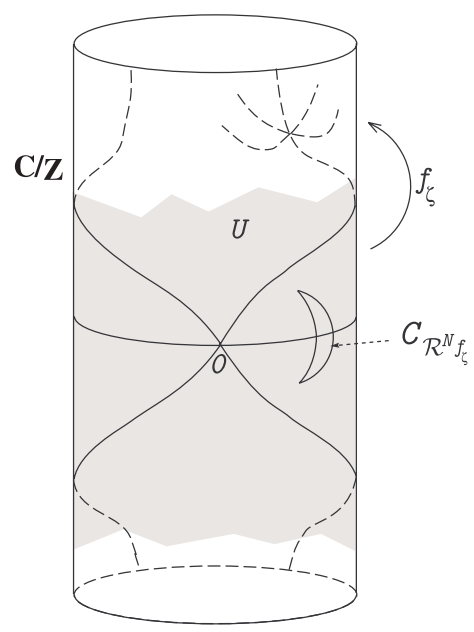

FIG. 4.

7.3. The cylinder renormalization operator

Proposition 7.9. - For every $s \in(0,1)$ there exists an equatorial neighborhood $\mathrm{U}=$ $\mathrm{U}(s)$ such that for every commuting pair $\zeta \in \mathscr{E}_{s}$ with $\chi(\zeta) \neq \infty$ the cylinder map $f_{\zeta}$ is in $\mathfrak{C}_{\mathrm{U}}$. 
Proof. - First, denote $\bmod \zeta=\sup \bmod U$ over all equatorial annuli $U$ such that $f_{\zeta} \in \mathfrak{C}_{\mathrm{U}}$. To prove the claim, let us assume the contrary. Then there is a sequence $\zeta_{n} \in \mathscr{E}_{s}$ with $\chi\left(\zeta_{n}\right) \neq \infty$ such that $\bmod \left(\zeta_{n}\right) \rightarrow 0$. Using compactness of $\mathscr{E}_{s}$ as stated in Lemma 3.2 we may pass to a subsequence $\zeta_{n} \rightarrow \zeta \in \mathscr{E}_{s}$. The height of the commuting pair $\zeta$ is necessarily infinite. The dynamics of $\zeta$ produces an analytic return map $h_{\zeta}$ from an equatorial neighborhood in the repelling Fatou cylinder of $\eta$ to the attracting Fatou cylinder. Again by Lemma 3.2, there exists $\delta=\delta(s)>0$ such that $h_{\zeta} \in \mathfrak{C}_{\mathrm{U}}$ with $\bmod \mathrm{U}>\delta$. By Theorem 6.4 for any $\zeta_{n}$ sufficiently close to $\zeta, \bmod \zeta_{n}>\delta$, and we have arrived to a contradiction.

For the remainder of this paper let us fix the value of $s$ to be the universal constant from Lemma 3.4. Let us say that two pairs $\zeta_{1}, \zeta_{2}$ in $\mathscr{E}_{s}$ are conformally equivalent, $\zeta_{1} \underset{\text { conf }}{\sim} \zeta_{2}$, if $\chi\left(\zeta_{1}\right)=\chi\left(\zeta_{2}\right) \neq \infty$ and $\left.\left.f_{\zeta_{1}}\right|_{\mathbf{T}} \equiv f_{\zeta_{2}}\right|_{\mathbf{T}}$. The name of the equivalence relation is explained by the following:

Proposition 7.10. - Suppose $\zeta_{1} \underset{\text { conf }}{\sim} \zeta_{2}$. Then there exists a conformal map $\psi$ from a neighborhood of $\mathrm{I}_{\zeta_{1}}$ to a neighborhood of $\mathrm{I}_{\zeta_{2}}$ which conjugates the two Epstein commuting pairs. Conversely, assume that there is a conjugacy $\psi$, whose domain includes a fundamental crescent $\mathrm{C}_{\zeta_{1}}$. Then $\zeta_{1} \underset{\text { conf }}{\sim} \zeta_{2}$.

Proof. - The first claim is quite obvious, since the identity map of $\mathbf{C} / \mathbf{Z}$ lifts via the projections $\pi_{\mathrm{C}_{\zeta_{1}}}, \pi_{\mathrm{C}_{\zeta_{2}}}$ to a conformal conjugacy between the two commuting pairs. On the other hand, any such conjugacy $\psi$ pojects to a conformal map $h$ between equatorial annuli, which conjugates $f_{\zeta_{1}}, f_{\zeta_{2}}$. If the domain of $\psi$ contains $\mathrm{C}_{\zeta_{1}}$, then $h$ extends to a conformal endomorphism of $\mathbf{G} / \mathbf{Z}$. Since $h(0)=0$, in this case $h \equiv \mathrm{Id}$.

Recall that $\mathfrak{S}_{r}=\{\zeta \in \mathfrak{C} \mid \chi(\zeta)=r\}$. The equivalence relation $\widetilde{c}$ is is clearly closed. By Lemma 7.6 we have:

Proposition 7.11. - For every $r \in \mathbf{N}$ the correspondence $\iota:\left(\mathfrak{S}_{r} \cap \mathscr{E}_{s}\right) / \widetilde{c o n f} \rightarrow \mathfrak{C}_{\mathrm{U}}^{\mathbf{R}}$ given by $[\zeta]_{\text {conf }}^{\sim} \mapsto f_{\zeta}$ is continuous.

Moreover,

Proposition 7.12. - For every $s \in(0,1)$ and $r \in \mathbf{N}$ there exists $c=c(s, r)>0$ such that the following holds. If we equip the Epstein class $\mathscr{E}_{S}$ with $\mathrm{C}^{0}$-distance, and $\mathfrak{C}_{\mathrm{U}}^{\mathbf{R}}$ with the sup-distance, then for every $\zeta_{1}, \zeta_{2} \in \mathscr{E}_{s} \cap \mathfrak{S}_{r}$ we have $\operatorname{dist}_{\mathrm{C}^{0}}\left(\zeta_{1}, \zeta_{2}\right) \geq c(s, r) \operatorname{dist}_{\mathrm{C}^{0}}\left(f_{\zeta_{1}}, f_{\zeta_{2}}\right)$.

Proof. - For $\zeta=(\eta, \xi) \in \mathscr{E}_{s}$ let $\phi$ denote the uniformizing coordinate $\mathrm{C}_{\zeta} \rightarrow \mathbf{G} / \mathbf{Z}$, and $\Phi: \mathrm{C}_{\zeta} \rightarrow \mathbf{G}$ be its lift. By Lemma 7.6 the crescent $\mathrm{C}_{\zeta}$ moves continuously in $\mathscr{E}$. On the other hand, by Lemma 3.2, $\mathscr{E}_{s}$ is Carathéodory compact, and hence the domain $\overline{\mathrm{C}}_{\zeta} \cup \eta\left(\mathrm{C}_{\zeta}\right) \cup \eta^{-1}\left(\mathrm{C}_{\zeta}\right)$ has a bounded shape near 
$\mathrm{C}_{\zeta} \cap \mathbf{R}$. Since $\Phi$ conformally extends to $\overline{\mathrm{C}}_{\zeta} \cup \eta\left(\mathrm{C}_{\zeta}\right) \cup \eta^{-1}\left(\mathrm{C}_{\zeta}\right)$, we may apply the Koebe Distortion Theorem to it to conclude that the distortion of $\Phi$ on $\mathrm{C}_{\zeta} \cap \mathbf{R}$ is bounded by a constant which depends only on $s$ and $r$. Moreover, by real a priori bounds the derivative $\Phi^{\prime}$ is bounded by const $(s, r)$. Noting that $\left.f_{\zeta}\right|_{\mathbf{T}}$ is the projection of the composition $\eta^{r} \circ \xi$, we have the desired estimate.

Proposition 7.13. - Let $\mathrm{U}$ be as in Proposition 7.9. There exists $\mathrm{N}=\mathrm{N}(\mathrm{U}) \in \mathbf{N}$ such that the following holds:

(I) suppose $\zeta \in \mathscr{E}_{s}$ is at least $\mathrm{N}$-times renormalizable. Then the renormalization $\mathscr{R}^{\mathrm{N}} \zeta \in \mathscr{E}_{s}$

(II) also $\mathscr{R}^{\mathrm{N}} \zeta$ has a holomorphic pair extension $\mathscr{H}$ with universal bounds as in Theorem 5.4;

(III) denote $\mathscr{H}^{\prime}$ the holomorphic pair extension of $p^{\mathrm{N}} \zeta$ which is the linear rescaling of $\mathscr{H}$. Then there exists an iterate $\eta^{-l}$ which conformally conjugates the pair $\mathscr{H}^{\prime}$ to a holomorphic pair $\mathscr{G}$ with $\Delta_{\mathscr{G}} \Subset \mathrm{C}_{\zeta}$ for some fundamental crescent $\mathrm{C}_{\zeta}$;

(IV) moreover, $\pi_{\mathrm{C}_{\zeta}}: \Delta_{\mathscr{G}} \rightarrow \mathbf{G} / \mathbf{Z} \cap \mathrm{U}$;

(V) finally, there is a fundamental crescent $\mathrm{C}_{p \mathscr{R}^{\mathrm{N}-1}} f_{5}$ compactly contained in $\mathrm{U}$ (cf. Figure 4).

Proof. - By real a priori bounds, eventually all renormalizations of a commuting pair $\zeta \in \mathscr{E}$ lie in $\mathscr{E}_{s}$. The existense of $\mathrm{N}$ as in (I) follows from Lemma 3.2. Statement (II) is a consequence of Theorem 5.4 and Lemma 3.2. Claims (III) and (IV) follow since the range of the holomorphic pair $\mathscr{H}^{\prime}$ is commensurable with $\mathrm{I}_{\mathrm{N}}$ by Theorem 5.4. Finally, (V) follows from Lemma 7.6 and Lemma 3.2.

Definition 7.2. - For the remainder of the paper fix $\mathrm{U}$ as in Proposition 7.9 and set $\mathfrak{M}=\mathfrak{C}_{\mathrm{U}}^{\mathbf{R}}, \mathrm{N}=\mathrm{N}(\mathrm{U})$. Condition $(V)$ of Proposition 7.13 implies that $f_{\zeta}$ is a renormalizable critical cylinder map. Let $\hat{f}$ be its renormalization corresponding to the crescent $\mathrm{C}_{p \mathscr{R}^{\mathrm{N}-1}} f_{\zeta}$. We will call it the cylinder renormalization of $f_{\zeta}$, and write

$$
\hat{f} \equiv \mathscr{R}_{\text {cyl }} f_{\zeta} .
$$

It followes from the first condition of Proposition 7.13 that $\mathscr{R}_{\mathrm{cyl}} f_{\zeta} \in \mathfrak{C}_{\mathrm{U}}$. Therefore, by Lemma 7.3, for every pair $\zeta$ as above, the tranformation $f_{\zeta} \mapsto \mathscr{R}_{\text {cyl }} f_{\zeta}$ extends to an open neighborhood $\mathrm{Y} \subset \mathfrak{C}_{\mathrm{U}}$ as an analytic operator $\mathrm{Y} \rightarrow \mathfrak{C}_{\mathrm{U}}$. We shall call this operator the cylinder renormalization operator.

The definition of $\mathscr{R}_{\text {cyl }}$ implies the following connection with the usual renormalization operator:

Proposition 7.14. - In the above notation, we have

$$
\mathscr{R}_{\mathrm{cyl}} f_{\zeta} \equiv f_{\mathscr{R}^{\mathrm{N}} \zeta}
$$


Proof. - By condition (V) of Proposition 7.13, there is an iterate $f^{\operatorname{tqN}}$, for some $t \in \mathbf{Z}$, which conformally maps $\pi_{\mathrm{C}_{\zeta}}^{-1}\left(\mathrm{C}_{\mathscr{R}^{N-1} f_{\zeta}}\right)$ to $\mathrm{C}_{\mathscr{R}^{N} \zeta}$. This conformal map projects to a conjugacy $\psi: \mathbf{C} / \mathbf{Z} \rightarrow \mathbf{G} / \mathbf{Z}$ between $\mathscr{R}_{\text {cyl }} f_{\zeta}$ and $f_{\mathscr{R}^{\mathrm{N}} \zeta}$. Since $\psi$ is conformal, and $\psi(0)=0$, necessarily $\psi \equiv$ Id.

Moreover,

Proposition 7.15. - If $\zeta_{1}, \zeta_{2}$ are at least $\mathrm{N}$-times renormalizable elements of $\mathscr{E}_{s}$, and $\zeta_{1} \underset{\text { conf }}{\sim} \zeta_{2}$, then $\mathscr{R}^{\mathrm{N}} \zeta_{1} \underset{\text { conf }}{\sim} \mathscr{R}^{\mathrm{N}} \zeta_{2}$. Hence the action of $\mathscr{R}^{\mathrm{N}}$ is well-defined on the quotient space $\mathscr{E}_{s} / \underset{\text { conf }}{\sim}$, and

$$
\iota \circ \mathscr{R}^{\mathrm{N}}=\mathscr{R}_{\mathrm{cyl}} \circ \iota \text {. }
$$

Proof. - The claim follows from condition (V) of Proposition 7.13, similarly to the preceding Proposition.

\section{Local stable manifold of a periodic point of $\mathscr{R}_{\mathrm{cyl}}$}

Let a commuting pair $\zeta$ be a periodic point of the renormalization operator $\mathscr{R}$. As follows from Lemma $3.4, \zeta \in \mathscr{E}_{s}$. Naturally, $\zeta$ is also a periodic point of $\mathscr{R}^{\mathrm{N}}$, and we may fix the smallest $m \in \mathbf{N}$ such that $\mathscr{R}^{\mathrm{N} m}(\zeta)=\zeta$. As seen from Proposition 7.15, the critical circle map $\hat{f}=f_{\zeta} \in \mathfrak{M}$ is a periodic point of the cylinder renormalization operator:

$$
\mathscr{R}_{\text {cyl }}^{m} \hat{f}=\hat{f} .
$$

Let us fix a periodic point $\hat{f}$. Set $\rho=\rho(\hat{f})$, and define $\mathscr{D}_{\rho}=\{f \in \mathfrak{M}$, such that $\rho(f)=\rho$. Again from Proposition 7.15 we have

Proposition 8.1. - There exists a neighborhood $\mathrm{Y}$ of $\hat{f}$ in $\mathfrak{M}$ such that for every $f \in \mathrm{Y} \cap \mathscr{D}_{\rho}, \mathscr{R}_{\text {cyl }}^{\text {im }} f$ is defined for all $i$, and $\mathscr{R}_{\text {cyl }}^{\text {im }} f \rightarrow \hat{f}$ uniformly in $\mathrm{Y}$.

Proof. - Assume that the statement of the proposition does not hold. Since $\mathscr{R}_{\text {cyl }}^{m}$ is defined in an open neighborhood of $\hat{f}$, the assumption implies that there exists $\delta>0$ such that for every $i \in \mathbf{N}$ and every $\epsilon>0$ there exists $f_{i, \epsilon}$ such that $\operatorname{dist}\left(\hat{f}, f_{i, \epsilon}\right)<\epsilon$, and $\operatorname{dist}\left(\hat{f}, \mathscr{R}_{\mathrm{cyl}}^{\text {im }} f_{i, \epsilon}\right)>\delta$. Select a sequence $\epsilon_{i} \rightarrow 0$ and denote $f_{i}=f_{i, \epsilon_{i}}$. Let $\lambda_{i}$ be the length of the domain of $(i m \mathrm{~N}-1)$-st pre-renormalization of $f_{i}: \lambda_{i}=\left|\mathrm{I}_{p \mathscr{R}^{i m \mathrm{~N}-1} f_{i}}\right|$, and consider the infinite sequence

$$
\mathscr{T}_{i}=\left(\lambda_{i}^{-1} p \mathscr{R}^{i m \mathrm{~N}-1+j} f_{i}\left(\lambda_{i} z\right)\right)_{j=-i m \mathrm{~N}-1}^{\infty} .
$$

By complex a priori bounds (Theorem 5.4) for each $i$ there exists such $\mathrm{N}$ that for all $n \geq \mathrm{N}$ the renormalization $\mathscr{R}^{n} f_{i}$ extends to a holomorphic commuting pair in 
$\mathfrak{H}(\mu)$ with a universal bound $\mu$. By Remark 5.1, the value of $\mathrm{N}$ can be chosen uniformly for all $i$. By Lemma 5.3 we may select a subsequence $\left\{i_{k}\right\}$ such that the sequences $\mathscr{T}_{i_{k}}$ converge to a limiting renormalization tower $\mathscr{T}$ with complex a priori bounds. Denote $\hat{\mathscr{T}}$ the limiting renormalization tower corresponding to the periodic point $\zeta$. By Proposition 7.12 , the distance between the base pairs of $\hat{\mathscr{T}}$ and $\mathscr{T}$ is at least const $\delta$. This contradicts the Tower Rigidity Theorem.

Below we shall demonstrate that the local stable set of $\hat{f}$ is a submanifold of $\mathfrak{M}$ :

Theorem 8.2. - There is an open neighborhood $\mathrm{W} \subset \mathfrak{M}$ of $\hat{f}$ such that $\mathscr{D}_{\rho} \cap \mathrm{W}$ is a smooth submanifold of $\mathfrak{M}$ of codimension 1 .

Fix an equatorial neighborhood $\mathrm{U}_{\hat{f}} \ni \mathrm{U}$ such that $\hat{f}$ analytically extends to $\mathrm{U}_{\hat{f}}$. As before denote $p_{k} / q_{k}$ the reduced form of the $k$-th continued fraction convergent of $\rho$. Furthermore, define $\mathscr{D}_{k}$ as the set of $g \in \mathfrak{M}$ for which $\rho(g)=p_{k} / q_{k}$ and 0 is a periodic point with period $q_{k}$. As follows from the Implicit Function Theorem, this is a codimension 1 submanifold of $\mathfrak{M}$. As seen in the previous section, the tangent space of a point in $\mathfrak{C}_{U}$ may be identified with $\mathfrak{B}_{U}$, and that of a point in $\mathfrak{M}$ with the real slice $\mathfrak{B}_{\mathrm{U}}^{\mathbf{R}}$. Let us define a cone $\mathscr{C} \in \mathfrak{B}_{\mathrm{U}}^{\mathbf{R}}$ as

$$
\mathscr{C}=\left\{v \in \mathfrak{B}_{\mathrm{U}}^{\mathbf{R}} \text {, such that } \inf _{x \in \mathbf{R}} v(x)>0\right\} .
$$

Lemma 8.3. - Let $f \in \mathscr{D}_{k}$, and denote $\mathrm{T}_{f} \mathscr{D}_{k} \subset \mathfrak{B}_{\mathrm{U}}^{\mathbf{R}}$ the tangent space at this point. Then $\mathrm{T}_{f} \mathscr{D}_{k} \cap \mathscr{C}=\emptyset$. that

Proof. - Let $v \in \mathscr{C}$ and suppose $\left\{f_{t}\right\} \subset \mathfrak{M}$ is a one-parameter family such

$$
\pi^{-1} \circ f_{t} \circ \pi=\pi^{-1} \circ f \circ \pi+t v+o(t) .
$$

Then for sufficiently small values of $t, \pi^{-1} \circ f_{t} \circ \pi>\pi^{-1} \circ f \circ \pi$. Hence $f_{t}^{q_{k}}(0) \neq f^{q_{k}}(0)$ and thus $f_{t} \notin \mathscr{D}_{k}$.

Now let $\hat{f}$ be as above. Elementary considerations of the Intermediate Value Theorem imply that for every $k$ there exists a value of $\theta \in(0,1)$ such that the map $f_{\theta}=\mathrm{R}_{\theta} \circ \hat{f} \in \mathscr{D}_{k}$. Moreover, if we denote $\theta_{k}$ the angle with the smallest absolute value satisfying this property, then $\theta_{k} \rightarrow 0$. Hence, for all $k$ large enough $\mathrm{R}_{\theta_{k}}\left(\mathrm{U}_{\hat{f}}\right) \supset \overline{\mathrm{U}}$, so $f_{\theta_{k}} \in \mathfrak{M}$. Set $f_{k}=f_{\theta_{k}}$ and let $\mathrm{T}_{k}=\mathrm{T}_{f_{k}} \mathscr{D}_{k} \subset \mathfrak{B}_{\mathrm{U}}^{\mathbf{R}}$. Fix $v \in \mathscr{C}$. By Lemma 8.3 and the Hahn-Banach Theorem there exists $\epsilon>0$ such that for every $k$ there exists a linear functional $h_{k} \in\left(\mathfrak{B}_{\mathrm{U}}^{\mathbf{R}}\right)^{*}$ with $\left\|h_{k}\right\|=1$, such that $\operatorname{Ker} h_{k}=\mathrm{T}_{k}$ 
and $h_{k}(v)>\epsilon$. By the Alaoglu Theorem, we may select a subsequence $h_{n_{k}}$ weakly converging to $h \in\left(\mathfrak{B}_{\mathrm{U}}^{\mathbf{R}}\right)^{*}$. Necessarily $v \notin \operatorname{Ker} h$, so $h \not \equiv 0$. Set $\mathrm{T}=\operatorname{Ker} h$.

Proof of Theorem 8.2. - By the above, we may select a splitting $\mathfrak{B}_{\mathrm{U}}^{\mathbf{R}}=\mathrm{T} \oplus v \cdot \mathbf{R}$. Denote $p: \mathfrak{B}_{\mathrm{U}}^{\mathbf{R}} \rightarrow \mathrm{T}$ the corresponding projection, and let $\psi: \mathfrak{M} \rightarrow \mathfrak{B}_{\mathrm{U}}^{\mathbf{R}}$ be a local chart at $\hat{f}$. Lemma 8.3 together with the Mean Value Theorem imply that $p \circ \psi: \mathscr{D}_{k} \rightarrow \mathrm{T}$ is an isomorphism onto the image, and there exists an open neighborhood $\mathscr{U}$ of the origin in $\mathrm{T}$, such that $p \circ \psi\left(\mathscr{D}_{k}\right) \supset \mathscr{U}$. Since the graphs $\mathscr{D}_{k}$ are analytic, we may select a $\mathrm{C}^{1}$-converging subsequence $\mathscr{D}_{k_{j}}$ whose limit is a smooth graph $\mathrm{G}$ over $\mathscr{U}$. Necessarily, for every $g \in \mathrm{G}, \rho(g)=\rho$. As we have seen above, every point $g \in \mathscr{D}_{\rho}$ in a sufficiently small neighborhood of $\hat{f}$ is in $\mathrm{G}$, and thus $\mathrm{G}$ is an open neighborhood in $\mathscr{D}_{\rho}$.

\section{Proof of the main result}

As in the previous section, let $\hat{f}: \mathrm{U}_{\hat{f}} \rightarrow \mathbf{C} / \mathbf{Z}$, where $\mathrm{U}_{\hat{f}} \ni \mathrm{U}$, be a periodic point of $\mathscr{R}_{\text {cyl }}$ with period $m$. Set $\rho=\rho(\hat{f})$ and let $\mathrm{W}$ be as in Theorem 8.2. Denote $\mathrm{T} \equiv \mathrm{T}_{\hat{f}}\left(\mathscr{D}_{\rho} \cap \mathrm{W}\right)$ the tangent space to the codimension one submanifold at $\hat{f}$, and let $\mathscr{L}$ be the differential of $\mathscr{R}_{\text {cyl }}$ at $\hat{f}$ :

$$
\mathscr{L}=\mathrm{D}_{\hat{f}} \mathscr{R}_{\text {cyl }}^{m}: \mathfrak{B}_{\mathrm{U}}^{\mathbf{R}} \rightarrow \mathfrak{B}_{\mathrm{U}}^{\mathbf{R}}, \mathscr{L}: \mathrm{T} \rightarrow \mathrm{T}
$$

Recall that a continuous linear operator on a Banach space is called compact if it maps the closed unit ball of the space onto a compact set. This condition is equivalent to the image of every closed bounded set being compact.

Proposition 9.1. - The operator $\mathscr{L}=\mathrm{D}_{\hat{f}} \mathscr{R}_{\text {cyl }}^{m}: \mathfrak{B}_{\mathrm{U}}^{\mathbf{R}} \rightarrow \mathfrak{B}_{\mathrm{U}}^{\mathbf{R}}$ is compact.

Proof. - Denote $\mathrm{B}_{1}$ the unit ball in $\mathfrak{B}_{\mathrm{U}}^{\mathbf{R}}$ and let $v \in \mathrm{B}_{1}$. By definition of $\mathscr{R}_{\text {cyl }}$, the image $\mathscr{L}_{v}$ is an analytic vector field on $\mathrm{U}_{\hat{f}} \ni \overline{\mathrm{U}}$. Let $\mathrm{C}_{\hat{f}} \Subset \mathrm{U}$ be the fundamental crescent corresponding to $\mathscr{R}_{\text {cyl }} \hat{f}$, and $\phi=\pi_{\hat{f}}: \mathrm{C}_{\hat{f}} \rightarrow \mathbf{C} / \mathbf{Z}$ the uniformizing coordinate. The first return map $\mathrm{R}$ of $\phi^{-1}(\mathrm{U}) \subset \mathrm{C}_{\hat{f}}$ consists of a finite collection of iterates $\hat{f}^{j_{k}}$. By compactness considerations, the vector field $v_{j_{k}}$ which is the perturbation of each of these iterates induced by $v$ has norm bounded by a constant $\mathrm{C}$ independent of $v$. Since $\phi^{\prime}$ is also bounded in $\phi^{-1}(\mathrm{U})$, there exists $\mathrm{D}>0$ independent of $v$ such that $\left.|| \mathscr{L}_{v}\right|_{\mathrm{U}} \|<\mathrm{D}$. Since the analytic functions in $\mathrm{U}_{\hat{f}}$ whose norms in $\mathrm{U}$ are bounded by $\mathrm{D}$ form a normal family in $\mathrm{U}$, the image $\mathscr{L}\left(\mathrm{B}_{1}\right)$ is compact.

Hyperbolicity: stable direction. — By Proposition 8.1,

$$
\left.\sup _{k}|| \mathscr{L}^{k}\right|_{\mathrm{T}} \|<\infty .
$$


Let us show that for some iterate the norm is strictly less than one:

Proposition 9.2. - There exists $k \in \mathbf{N}$ such that $\left\|\left.\mathscr{L}^{k}\right|_{\mathrm{T}}\right\|<1$.

Proof. - Assume the contrary. Then the operator $\left.\mathscr{L}\right|_{\mathrm{T}}$ has a unit spectral radius. The discreteness of the spectrum of a compact operator implies that $\operatorname{Sp}\left(\left.\mathscr{L}\right|_{\mathrm{T}}\right) \cap \mathrm{B}_{1} \neq \varnothing$, and moreover, since every non-zero element of the spectrum of a compact operator is an eigenvalue, there is $v \in \mathrm{T}$ such that $\mathscr{L}(v)=v$. The space $\mathrm{T}$ may then be split into a direct sum $\mathrm{E}^{s} \oplus \mathrm{E}^{c}$ such that $\mathrm{E}^{s}$, the stable space, is a closed $\mathscr{L}$-invariant subspace such that $\left\|\left.\mathscr{L}^{\ell}\right|_{\mathrm{E}^{s}}\right\|<1$ for some $\ell \in \mathbf{N}$, and $\mathrm{E}^{c}$, the central space, is also $\mathscr{L}$-invariant, finite dimensional, and for every $v \in \mathrm{E}^{c}, \inf _{\ell \in \mathbf{N}}\left\|\mathscr{L}^{\ell} v\right\|>0$. The space $\mathrm{E}^{c}$ is spanned by solutions of $(\mathrm{T}-\lambda \mathrm{Id})^{k} v=0$, $k \in \mathbf{N}, \lambda \in \mathrm{B}_{1}$. If we choose a small perturbation $f \in \mathscr{D}_{\rho} \cap \mathrm{W}$ of $\hat{f}$ in the direction of $\mathrm{E}^{c}$ close enough to $\hat{f}$, then the dynamics of $\mathscr{R}_{\text {cyl }}^{m \ell}$ on $f$ is going to be dominated by the central part $\left.\mathscr{L}^{\ell}\right|_{\mathrm{E}^{c}}$. In particular, the rate of convergence $\mathscr{R}_{\text {cyl }}^{k m \ell} f \underset{k \rightarrow \infty}{\longrightarrow} \hat{f}$ can not be geometric, contrary to Theorem 4.3.

Hyperbolicity: unstable direction. - As before, let

$$
\mathscr{C}=\left\{v \in \mathfrak{B}_{\mathrm{U}}^{\mathbf{R}} \text {, such that } \inf _{x \in \mathbf{R}} v(x)>0\right\} .
$$

The key properties of $\mathscr{C}$ are listed in the next lemma:

\section{Lemma 9.3.}

(I) The cone $\mathscr{C}$ is renormalization-invariant: $\mathscr{L}: \mathscr{C} \rightarrow \mathscr{C}$,

(II) Moreover, there exists $\alpha>0$ and $k \in \mathbf{N}$ such that for any vector field $v \in \mathscr{C}$

$$
\inf _{x \in \mathbf{R}} \mathscr{L}^{k}(v(x))>(1+\alpha) \inf _{x \in \mathbf{R}} v(x),
$$

(III) Finally, if $v \in \mathfrak{B}_{\mathrm{U}}^{\mathbf{R}}$ belongs to the closure of $\mathscr{C}$, and $v \neq 0$, then there exists $\ell \in \mathbf{N}$ for which $\mathscr{L}^{\ell}(v) \in \mathscr{C}$.

Proof. - Let $\tilde{f}=\pi^{-1} \circ \hat{f} \circ \pi$. As follows from an elementary computation, if $\tilde{f}_{t}(x)=\tilde{f}(x)+t v(x)+o(t)$, then

$$
\begin{aligned}
\tilde{f}_{t}^{2}(x) & =\tilde{f}^{2}(x)+t v_{2}(x)+o(t) \\
& =\tilde{f}^{2}(x)+t\left(\tilde{f}^{\prime}(\tilde{f}(x)) v(x)+v(\tilde{f}(x))\right)+o(t),
\end{aligned}
$$

and more generally, if we write

$$
\begin{aligned}
& \tilde{f}_{t}^{n}(x)=\tilde{f}^{n}(x)+t v_{n}(x)+o(t), \\
& \text { then } v_{n}(x)=f^{\prime}\left(f^{n-1}(x)\right) v_{n-1}(x)+v\left(f^{n-1}(x)\right) .
\end{aligned}
$$


Let us denote $\mathrm{C}_{k} \Subset \mathrm{U}$ the fundamental crescent of $f$ corresponding to the $k$-th cylinder renormalization $\mathscr{R}_{\text {cyl }}^{k} f$, and $\mathrm{J}_{k}=\mathrm{C}_{k} \cap \mathbf{T}$. Let $\pi_{k}(x)$ be the corresponding uniformizing coordinate $\mathbf{C}_{k} \rightarrow \mathbf{G} / \mathbf{Z}$, and $\Phi_{k}: \mathbf{C}_{n} \rightarrow \mathbf{G}$ its lift. In the local chart given by $\pi_{k}^{-1}$, the $k$-th cylinder renormalization $\mathscr{R}_{\text {cyl }}^{k} f$ is represented by the iterate $g_{k}=f^{q_{m k}}$ for some $m \in \mathbf{N}$.

To prove the first claim, observe that by (9.2),

$$
\inf _{x \in \mathbf{R}} v_{n}(x) \geq \inf _{x \in \mathbf{R}} v(x)>0 .
$$

The image

$$
\mathscr{L}_{v}=\left[\left(\Phi_{k}\right)^{\prime} \circ g_{k} \cdot\left(\left.v_{q_{m k}}\right|_{\mathrm{J} k}\right)\right] \circ\left(\Phi_{k}\right)^{-1} .
$$

Since $\left(\Phi_{k}\right)^{\prime}>0$, (I) follows.

To prove (II), note that by the real a priori bounds, the sizes of the intervals $\mathrm{J}_{k}$ decrease geometrically with $k$. On the other hand, applying the Koebe Distortion Theorem to the conformal extension of $\Phi_{k}$ to $\mathrm{C}_{k}$ and its two neighboring iterates, we see that the distortion of $\Phi_{k}$ on the interval $g_{k}\left(\mathrm{~J}_{k}\right)$ is bounded uniformly in $k$. Hence, there exists $\alpha>0$ and $l \in \mathbf{N}$ such that for all $k \geq l$

$$
\left.\left(\Phi_{k}\right)^{\prime}\right|_{g_{k}\left(\mathrm{~J}_{k}\right)}>1+\alpha
$$

and (II) follows from (9.4).

Finally, to see (III), observe that if $v(x) \not \equiv 0$, then there exist $\ell, n \leq q_{m \ell}$ such that $v(x)>0$ for every $x \in f^{n}\left(\mathrm{~J}_{\ell}\right)$. The claim now follows from (9.2).

We conclude:

Proposition 9.4. - There exists an eigenvector $\hat{v} \in \mathscr{C}$ :

$$
\mathscr{L}(\hat{v})=\delta \hat{v} \text {, where }|\delta|>1 \text {. }
$$

Proof. - Part (II) of the previous Lemma implies that the spectral radius $\mathrm{R}_{\mathrm{Sp}}(\mathscr{L})>1$. Since the spectrum of a compact operator is discrete, and its every non-zero element is an eigenvalue, the claim follows.

Universality: Proof of Corollary 4.5. - The second claim is an obvious corollary of the main theorem, the proof of the first one follows. Denote $\zeta_{*} \in \mathscr{E}_{s}$ the fixed point of $\mathscr{R}$ with $\rho\left(\zeta_{*}\right)=(\sqrt{5}-1) / 2$, and $\operatorname{set} f_{*}=\iota\left(\zeta_{*}\right)$, defined in some annulus $\mathrm{U}_{f_{*}} \supset \overline{\mathrm{U}}$. By Theorem 4.4 , there exists $m \in \mathbf{N}$ such that $f_{*}$ is a hyperbolic fixed point of $\mathscr{R}_{\text {cyl }}$ with one-dimensional unstable direction. Let $\mathrm{W}_{s}^{\text {loc }}$ be its local stable manifold, set $\mathrm{M}=m \mathrm{~N}$, and denote $\delta^{\mathrm{M}}>1$ the modulus of the unstable eigenvalue. Set

$$
\begin{aligned}
\mathfrak{P}_{n}^{ \pm}= & \{f \in \mathfrak{C}_{\mathrm{U}}, \text { such that } \rho(f)=\overbrace{[1, \ldots, 1]}^{n}, \\
& \text { and } f \text { has a periodic orbit with eigenvalue } \pm 1\} .
\end{aligned}
$$


By the Implicit Function Theorem, $\mathfrak{P}_{n}^{ \pm}$is a local submanifold of $\mathfrak{C}_{\mathrm{U}}$ of codimension one. Moreover, in a neighborhood of $f_{*}, \mathscr{R}_{\text {cyl }}: \mathfrak{P}_{n}^{ \pm} \rightarrow \mathfrak{P}_{n-\mathrm{M}}^{ \pm}$. Let $\mathscr{C}^{\prime}$ be a subcone of $\mathscr{C}$ with $\mathscr{C}^{\prime} \cap \mathrm{B}_{1}$ compact. Then by Theorem 4.4, there exists an open neighborhood $\mathrm{W} \subset \mathfrak{C}_{\mathrm{U}}$ of $f_{*}$ and $\mathrm{K} \in \mathbf{N}$, such that for $k>\mathrm{K}, \mathfrak{P}_{k}^{ \pm} \cap \mathrm{W}$ is a graph over $\mathrm{W}_{s}^{\text {loc }}$ transversal to $\mathscr{C}^{\prime}$ at every point. Moreover, for every $n>\mathrm{K}$ the sequence $\left\{\mathfrak{P}_{n+l \mathrm{M}}^{ \pm}\right\}_{l=1}^{\infty}$ converges to $\mathrm{W}_{s}^{\text {loc }}$ at a geometric rate $\delta^{\mathrm{M}}$. For our purposes we will set $\mathscr{C}^{\prime}=\mathrm{D}_{f_{*}} \mathscr{R}_{\text {cyl }}^{m}(\mathscr{C})$.

Let $\mathrm{V} \supset \mathbf{T}$ be an equatorial annulus chosen so that $f_{\mu_{*}} \in \mathfrak{C}_{\mathrm{V}}$. By compex a priori bounds, there exists $\mathrm{N}_{1} \in \mathbf{N}$ such that for all $n>\mathrm{N}_{1}$ the renormalization $\mathscr{R}^{n} f_{\mu_{*}}$ extends to a holomorphic commuting pair with universal bounds. Since $\mathscr{R}^{n} f_{\mu_{*}} \rightarrow \zeta_{*} \in \mathscr{E}_{s}$ and by Lemma 7.6, there exists $\mathrm{N}_{2} \geq \mathrm{N}_{1}$ such that for all $k>\mathrm{N}_{2}$ the map $f_{\mu_{*}} \in \mathfrak{C}_{\mathrm{V}}$ is cylinder-renormalizable with period $q_{k}$, and the corresponding cylinder renormalization $g_{k}$ is in $\mathfrak{C}_{\mathrm{U}}$. Fix some such $k$. By Proposition 7.3, there is a neighborhood $\mathrm{G}$ of $f_{\mu_{*}}$ in $\mathfrak{C}_{\mathrm{V}}$ such that the cylinder renormalization $f_{\mu_{*}} \mapsto g_{k}$ extends to an analytic operator $\mathrm{Z}: \mathrm{G} \rightarrow \mathfrak{C}_{\mathrm{U}}$. Provided $\mathrm{G}$ is sufficiently small, there is an iterate $\mathscr{R}_{\text {cyl }}^{t}(\mathrm{G}) \subset \mathrm{W}$. Denote $\left\{h_{\mu}\right\} \subset \mathfrak{C}_{\mathrm{U}}$ the smooth family $\mathscr{R}_{\text {cyl }}^{t}\left(\mathrm{Z}\left(\left\{f_{\mu}\right\} \cap \mathrm{G}\right)\right)$. Let $u \in \mathfrak{B}_{\mathrm{V}}^{\mathbf{R}}$ be the tangent vector to the family $f_{\mu}$ at $f_{\mu_{*}}$, and $v \in \mathfrak{B}_{\mathrm{U}}^{\mathbf{R}}$ its image $\mathrm{D}_{f_{\mu_{*}}}\left(\mathscr{R}_{\mathrm{cyl}}^{t} \circ \mathrm{Z}\right) u$. As in Lemma 9.3 we have inf $\mathrm{D}_{f_{\mu_{*}}} \mathrm{Z} u>0$ and hence $v \in \mathscr{C}^{\prime}$. Note that the curve $\left\{h_{\mu}\right.$ for $\left.\mu \in \mathrm{I}_{i+l \mathrm{M}}\right\}$ for $i$ large is bounded by a pair of points $f_{n}^{ \pm} \in \mathfrak{P}_{n}^{ \pm}$, where $n=i+l \mathbf{M}-k-t \mathbf{M}$, and the claim follows from the transversality property of $\mathfrak{P}_{n}^{ \pm}$.

\section{Concluding remarks}

Let us conclude by discussing some remaining open problems in the field. Of primary importance to us is bringing Lanford's Program to a completion. Passing from the hyperbolicity of periodic orbits of $\mathscr{R}_{\text {cyl }}$ to the hyperbolicity of the whole renormalization horseshoe requires, as a preliminary step, endowing the local stable sets of the points in the horseshoe with the structure of analytic submanifolds. In addition, the case of unbounded type necessitates analyzing the situation when the periods of renormalization grow without a bound, and showing that the rate of convergence to the horseshoe remains uniformly bounded in this situation. We recently carried out the relevant analysis in a joint paper with A. Epstein $[\mathrm{EY}]$. We extend the result of this paper to the whole horseshoe in a forthcoming work [Ya3].

Commonly, renormalization convergence results in one-dimensional dynamics

are connected with rigidity properties of the renormalized maps. The problem relevant to our study is a $\mathrm{C}^{1+\alpha}$ rigidity question:

When is the conjugacy between two critical circle maps with the same irrational rotation number, which maps 0 to $0, \mathrm{C}^{1+\alpha}$-smooth? 
The study of this question is the main subject of the papers of de Faria and de Melo [dFdM1,dFdM2]. These authors demonstrate that the conjugacy has the desired smoothness in the case when both maps are analytic, and the type of the rotation number is bounded. They prove that, with these restrictions, the $\mathrm{C}^{1+\alpha}$ rigidity is equivalent to the geometric rate of convergence of renormalizations, and derive their result from their Theorem 4.3. On the other hand, they show that if both of the conditions are relaxed, the statement does not hold. As we described above, we expect our methods to bring Lanford's Program to a completion, and in particular, to extend Theorem 4.3 to unbounded types. It is then an intriguing problem, whether the answer to the above question is positive when the two maps are analytic, but the rotation numbers are unbounded. The situation when the type is bounded, but the maps are only smooth also requires study.

In this paper we have introduced a new renormalization for critical circle maps. We hope that the reader has been convinced that apart from enabling us to solve the hyperbolicity problem, this new construction is more natural. It would be instructive therefore to re-do the proof of Theorem 4.1 using $\mathscr{R}_{\text {cyl }}$ instead of $\mathscr{R}$. This mainly requires understanding the geometry of a periodic point of $\mathscr{R}_{\text {cyl }}$ to define an appropriate analogue of the Epstein class, and finding the corresponding replacements of de Faria's holomorphic commuting pairs. For the moment, the only situation when we are able to carry out the whole theory without recourse to commuting pairs is the degenerate case of parabolic renormalization, studied in $[\mathrm{EY}]$.

Let us also comment that our method should be applicable to the study of universality of Siegel disks (see e.g. $[\mathrm{MP}]$ ) in the situation when complex a priori bounds exist (such as Siegel quadratics of bounded type, cf. [Ya1]). This problem is analogous to the study of renormalization of non-real quadratic polynomials and the related aspects of the geometry of the Mandelbrot set, and should lead to the construction of hyperbolic non-real periodic orbits of $\mathscr{R}_{\text {cyl }}$.

\section{REFERENCES}

[BR] L. Bers and H. L. Royden, Holomorphic families of injections, Acta Math., 157 (1986), 259-286.

[Do] A. Douady, Does a Julia set depend continuously on the polynomial?, in Complex dynamical systems: The mathematics behind the Mandelbrot set and Julia sets, R. L. Devaney (ed.), Proc. of Symposia in Applied Math., Vol. 49, Amer. Math. Soc., 1994, pp. 91-138.

[DH1] A. Douady and J. H. Hubbard, Etude dynamique des polynômes complexes, I-II, Pub. Math. d'Orsay, 1984.

[DH2] A. Douady and J. H. Hubbard, On the dynamics of polynomial-like mappings, Ann. Sci. Éc. Norm. Sup., 18 (1985), 287-343.

[dF1] E. DE FARIA, Proof of universality for critical circle mappings, Thesis, CUNY, 1992.

[dF2] E. DE FARIA, Asymptotic rigidity of scaling ratios for critical circle mappings, Ergodic Theory Dynam. Systems, 19 (1999), no. 4, 995-1035.

[dFdM1] E. de Faria and W. de Melo, Rigidity of critical circle mappings I, f. Eur. Math. Soc. (fEMS), 1 (1999), no. 4, 339-392. 
[dFdM2] E. De Faria and W. DE Melo, Rigidity of critical circle mappings II, f. Amer. Math. Soc., 13 (2000), no. 2, 343-370

[Ep1] A. Epstein, Towers of finite type complex analytic maps, PhD Thesis, CUNY, 1993.

[EKT] A. Epstein, L. Keen and C. Tresser, The set of maps $\mathrm{F}_{a, b}: x \mapsto x+a+\frac{b}{2 \pi} \sin (2 \pi x)$ with any given rotation interval is contractible, Commun. Math. Phys., 173 (1995), 313-333.

[EY] A. Epstein and M. Yampolsky, The universal parabolic map. Erg. Th. E Dyn. Systems, to appear.

[EE] J.-P. Eckmann and H. Epstein, On the existence of fixed points of the composition operator for circle maps, Commun. Math. Phys., 107 (1986), 213-231.

[FKS] M. Feigenbaum, L. Kadanoff, and S. Shenker, Quasi-periodicity in dissipative systems. A renormalization group analysis, Physica, 5D (1982), 370-386.

[He] M. Herman, Conjugaison quasi-symmetrique des homeomorphismes analytiques du cercle a des rotations, manuscript.

[Keen] L. KeEn, Dynamics of holomorphic self-maps of $\mathbf{G}^{*}$, in Holomorphic functions and moduli I, D. Drasin et al. (eds.), Springer-Verlag, New York, 1988.

[Lan1] O. E. LANFORD, Renormalization group methods for critical circle mappings with general rotation number, in VIIIth International Congress on Mathematical Physics (Marseille, 1986), pp. 532-536, World Sci. Publishing, Singapore, 1987.

[Lan2] O. E. LANFORD, Renormalization group methods for critical circle mappings, Nonlinear evolution and chaotic phenomena, NATO Adv. Sci. Inst. Ser. B: Phys., 176, pp. 25-36, Plenum, New York, 1988.

[Lyu2] M. Lyubich, Renormalization ideas in conformal dynamics, Cambridge Seminar "Current Developments in Math.”, May 1995, pp. 155-184, International Press, 1995, Cambridge, MA.

[Lyu3] M. Lyubich, Dynamics of quadratic polynomials, I-II, Acta Math., 178 (1997), 185-297.

[Lyu4] M. Lyubich, Feigenbaum-Coullet-Tresser Universality and Milnor's Hairiness Conjecture, Ann. of Math. (2), 149 (1999), no. 2, 319-420.

[Lyu5] M. Lyubich, Almost every real quadratic map is either regular or stochastic, Ann. of Math. (2), 156 (2002), no. $1,1-78$.

[LY] M. Lyubich and M. Yampolsky, Dynamics of quadratic polynomials: complex bounds for real maps, Ann. l'Inst. Fourier 47, 4 (1997), 1219-1255.

[MP] R. S. Mackay and I. C. Percival, Universal small-scale structure near the boundary of Siegel disks of arbitrary rotation number, Physica, 26D (1987), 193-202.

[MSS] R. Mañé, P. SAd and D. Sullivan, On the dynamics of rational maps, Ann. Sci. Éc. Norm. Sup., 16 (1983), 193-217.

[McM1] G. McMullen, Complex dynamics and renormalization, Annals of Math. Studies, v.135, Princeton Univ. Press, 1994.

[McM2] C. MaMullen, Renormalization and 3-manifolds which fiber over the circle, Annals of Math. Studies, Princeton University Press, 1996.

[Mes] B. D. Mestel, A computer assisted proof of universality for cubic critical maps of the circle with golden mean rotation number, PhD Thesis, University of Warwick, 1985.

[Mil] J. Milnor, Dynamics in one complex variable, Introductory lectures, Friedr. Vieweg \& Sohn, Braunschweig, 1999.

[MvS] W. De Melo and S. van Strien, One dimensional dynamics, Springer, 1993.

[ORSS] S. Ostlund, D. Rand, J. Sethna, and E. Siggia, Universal properties of the transition from quasiperiodicity to chaos in dissipative systems, Physica, 8D (1983), 303-342.

[Sul1] D. Sullivan, Quasiconformal homeomorphisms and dynamics, topology and geometry, Proc. ICM-86, Berkeley, v. II, 1216-1228.

[Sul2] D. Sullivan, Bounds, quadratic differentials, and renormalization conjectures, AMS Centennial Publications, 2 , Mathematics into Twenty-first Century (1992).

[Sh] M. Shishikura, The Hausdorff dimension of the boundary of the Mandelbrot set and Julia sets, Ann. of Math. (2), 147 (1998), no. 2, 225-267.

[Swl] G. Światek, Rational rotation numbers for maps of the circle, Commun. Math. Phys., 119 (1988), 109128.

[Yal] M. Yampolsky, Complex bounds for renormalization of critical circle maps, Erg. Th. E̊ Dyn. Systems, 19 (1999), 227-257. 
[Ya2] M. Yampolsky, The attractor of renormalization and rigidity of towers of critical circle maps, Commun. Math. Phys., 218 (2001), no. 3, 537-568.

[Ya3] M. Yampolsky, The global horseshoe for the renormalization of critical circle maps, Preprint, 2002.

[Yoc] J.-C. Yoccoz, Il n'ya pas de contre-example de Denjoy analytique, C.R. Acad. Sci. Paris, 298 (1984) série I, $141-144$

M. Y.

Department of Mathematics

University of Toronto

Toronto, Ontario M5S 3G3

Canada

yampol@math.utoronto.ca

Manuscrit reçu le 5 décembre 2000. 